\title{
Oral Communications
}

\section{TRAUMATOLOGY 1}

The role of Girdlestone resection arthroplasty in a case of posterior hip dislocation associated to femoral neck fracture, transverse acetabular fracture and sciatic nerve injury

\author{
R. Giancola, F. Valli, G. Antonini, C. Crippa \\ Department of Orthopaedic and Trauma Surgery, San Carlo \\ Borromeo Hospital (Milan, ITALY)
}

Dislocations of the hip have become a common injury in recent years and it can occur in conjunction with a fracture of the femoral shaft or neck or head. Posterior dislocation of the hip associated with femoral neck fracture along with fracture of the acetabulum but an intact femoral head is an unusual but not an unknown injury. This paper reports a patient who sustained posterior dislocation of the hip associated with fracture of the femoral neck of the ipsilateral femur, transverse with posterior wall acetabular fracture and sciatic nerve injury. Such injuries present a therapeutic dilemma. Controversy usually exists regarding primary arthroplasty versus open reduction and internal fixation of the femoral neck fracture, but in this case the authors have to be considered the acetabular fracture, where total hip replacement is rarely indicated in the acute phase. However the open reduction and internal fixation's technique presents an high risk, $20-30 \%$, of subsequent avascular necrosis and an high rate, $35 \%$, of reprise; posttraumatic arthritis has been demonstrated in $12-67 \%$ of the patients with acetabular fracture, even when the acetabular bone stock could be restored with minimal deformity, while the posttraumatic avascular necrosis of the femoral head following an acetabular fracture has been reported to range from 2 to $40 \%$. Our patient was managed within five hours from injury by open reduction and internal fixation of acetabular fractures and proximal femoral resection arthroplasty (Girdlestone's technique). After 6 months a total hip arthroplasty was performed. The patient at 4 years from trauma presents a full range of motion, no pain, a good integration of arthroplasty, no osteolysis sign, but a paralysis of external sciatic nerve.

The purpose of this case report is to suggest a new surgical approach for the treatment of this association of lesions, introducing the
Girdlestone procedure like an intermediate option before definitive total hip replacement. To our knowledge in the English-language literature there is not a similar treatment solution.

Management of fractures ipsilateral femur and pelvis: floating hip

$\underline{\text { R. Spagnolo }}{ }^{1}$, G. Fioretta ${ }^{1}$, S. Angelini ${ }^{2}$, P.G. Davini ${ }^{2}$

${ }^{1}$ Department of Orthopaedics and Traumatology, Ospedale di Desio (Milan, ITALY);

${ }^{2}$ Department of Orthopaedics and Traumatology, Ospedale di Empoli (Florence, ITALY)

Objective We review 5 cases of ipsilateral femur and pelvis fractures and describe associated lesions, treatment, results, and complications. Material and methods The lesion mechanism involved a street accident for all patients, car to car collision in 3 cases and running over in 2 cases. According to Liebergall et al., 2 patients were type A, 3 type $B$, no patient type $C$. The time of surgery was determined by the hemodynamic status of the patient. Results were evaluated according to the criteria of d'Aubignè and Postel modified by Matta et al.

Results None of the patients died. According to the criteria of d'Aubignè and Postel modified by Matta et al., results were excellent in 3 cases, good in 2.

Discussion Ipsilateral fractures of the femur and pelvis are complex lesions requiring specific management training, they are severe injury usually caused by high energy trauma. The acetabulum and pelvic ring are more commonly fractured together than either alone. The acetabular fractures were treated with open reduction and internal fixation (ORIF) in all cases. The patients with pelvic ring fractures were surgically stabilized with external fixation in emergency and subsequently with ORIF. In case of femur fractures, acetabular fractures were first treated, while in case of association of pelvis and femur fractures, femur was treated after pelvic stabilization.

Suggested readings

1. Burd TA, Hughes MS et al. (2008) The Floating Hip: Complications and Outcomes. The journal of Trauma 64:442-448

2. Liebergall $\mathrm{M}$ et al. (1992) The floating hip. Ipsilateral pelvic and femoral fractures. J Bone Joint Surg Br 74:93-100 


\section{TRAUMATOLOGY 2}

\section{Biomechanical properties of different pin types and configurations for percutaneous fixation of 2-part proximal humeral fractures}

D. Bonasia ${ }^{1}$, F. Castoldi ${ }^{1}$, R. Rossi ${ }^{1}$, A. Sankey ${ }^{2}$, F. Dettoni ${ }^{1}$, D. Blonna ${ }^{1}$ A. Amis ${ }^{3}$

${ }^{1}$ Ospedale Mauriziano Umberto I, Università degli Studi di Torino (Turin, ITALY);

${ }^{2}$ St. Mary Hospital (London, UK);

${ }^{3}$ Imperial College (London, UK)

Introduction In 2005 a new percutaneous device has been developed for the treatment of proximal humeral fractures. This device has 2 main features: (1) long thread wires, and (2) an external fixator to link the wires together. The goal of this study was to investigate the biomechanical properties of different wire constructs (changing the size and type of the wires, and combining them in different configurations) compared to the locking plate (Fig. 1).

Material and methods A two-part proximal humeral fracture was created in 72 fourth-generation Sawbones. There were 9 groups of 8 humeri each. Each group was fixed with one of 9 different methods (Fig. 1). An Instron 8800 servohydraulic tension-torsion material testing machine was used for: (1) Cyclic torsion test for stiffness (Nm/ deg of rotation) and maximum rotation (deg); (2) cyclic axial compression-plus-varus (20 degrees) bending test for stiffness $(\mathrm{N} / \mathrm{mm})$ and maximum displacement (mm); (3) ultimate load to failure in the axial compression-plus-varus bending mode. Statistical analysis: ANOVA and post-hoc analysis with Bonferroni correction Significance $p<0.05$.

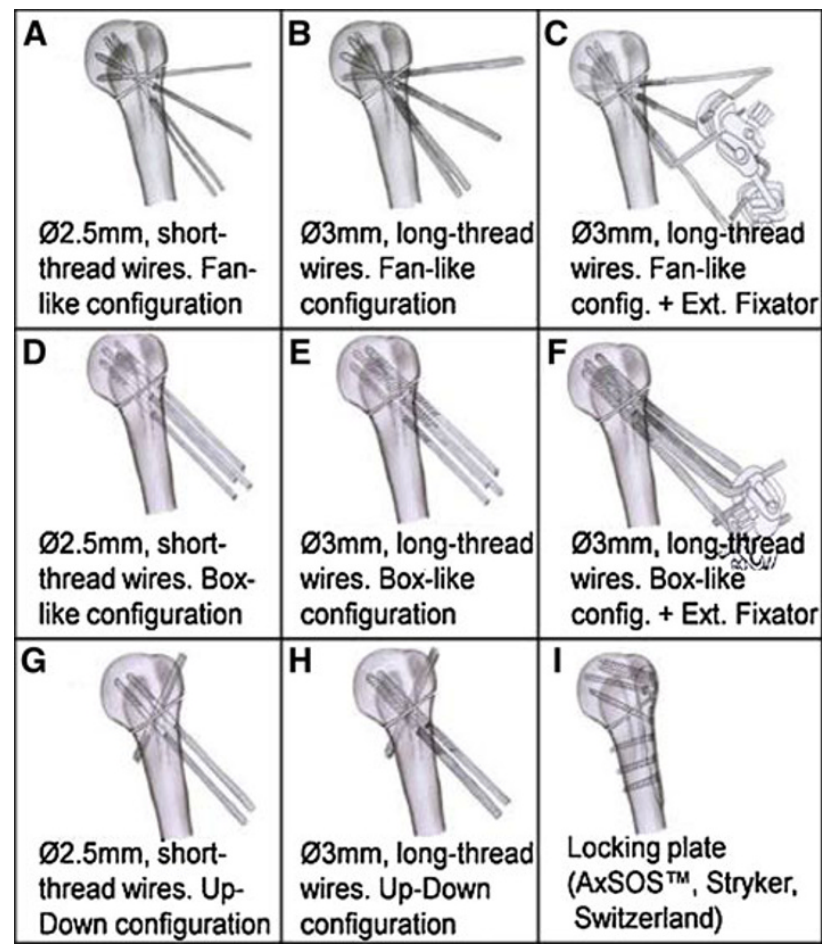

Fig. 1
Results 1. Stiffness: the external fixator tended to increase the torsional stiffness, but significantly only for the Box group $\left({ }^{*} p=0.03\right)$.The locking plate group had the highest torsional stiffness, but not significantly different from group $\mathrm{F}(* * p=1)$. Maximum rotation: data were similar to torsional stiffness. 2. Stiffness: the only construct significantly weaker $(p=0.03)$ was $\mathrm{H}$ when compared to $\mathrm{C}$ and $\mathrm{F}$. No other significant differences were found. $\mathrm{C}$ and $\mathrm{F}$ were stiffer than the Plate $(p=0.07)$. Maximum displacement: no differences were noted. 3 . With some constructs ( $8 / 8$ of group I, in $5 / 8$ of $\mathrm{F}$, and in $4 / 8$ of C), at $2000 \mathrm{~N}$, failure occurred at the shaft. The other constructs failed at fracture site at significantly lower loads. Discussion In group F, the properties did not differ significantly from the locking plate. Group $\mathrm{C}$ was comparable to $\mathrm{F}$ and $\mathrm{I}$ in every test, except in the torsion tests, where it showed inferior properties.

\section{TRAUMATOLOGY 3}

\section{The treatment of C2-3 distal humeral fractures in elderly patients with osteosynthesis (parallel-plate technique)}

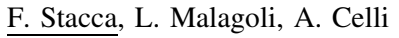

Clinica Ortopedica e Traumatologica, Policlinico di Modena (Modena, ITALY)

Introduction The gold standard in the treatment of C2-3 distal humeral fracture is the ORIF procedure. The target is the recovery of the anatomical complexity of the elbow with a strong stabilization, so to allow a early mobilization. This target is difficult in the adult, and more difficult in the elderly patient, who has a poor bone quality, so that an important part of the international literature indicates the arthroplasty as the primary treatment. Elbow prosthesis allows normal daily activities, but it could be a limitation in case of high functional requests. The aim of this study is the evaluation of the elderly patient with C2-3 distal humeral fractures treated in our clinic with parallel plates ORIF technique.

Material and methods In the period 2003-2009 we treated 14 patients over 65 with complex distal humeral fracture (AO:C2-3). All the patients had high functional requests. All the patients were treated with parallel plates (Mayo Clinic) as described by O'Driscoll. The evaluation interested all the patients: F.U. 29.2 months, main age:71.7 years, 6 male and 8 female. In 9 cases the fracture interested the dominant arm. Standard RX scan were used. Clinical evaluation followed standardized test: MEPS one and DASH one.

Results All the patients obtained a complete healing of the fracture. We observed good or excellent results in 13 patients, the main MEPS was 91.1 and main DASH was 17.5. R.O.M.: $18^{\circ}-131^{\circ}$ in flexionextension, $74^{\circ}-73^{\circ}$ in prono-supination. Pain was absent in 10 patients. Only one patient did not have a satisfactory outcome because of articular stiffness: there was the presence of many comorbidities and he couldn't attempt at a correct rehabilitation.

Discussion and conclusions The recovery of the anatomy is the first step in the treatment of $\mathrm{C} 2-3$ distal humeral fractures. A strong stabilization is also very important, so to allow the mobilization and avoid articular stiffness. In our experience we observed that the use of parallel plates system helps the surgeon for a correct reduction of the fracture, and can give a great stability of the fragments also in elderly patients with a poor bone quality. In conclusion it can represent the first choice also in over 65 patients, especially with high functional requests. 


\section{Treatment of fractures of the humerus diaphysis with Seidel endo-medullar pins}

\author{
L. Nalbone, G. Alongi, N. Galvano, G. Milicia, M. D’Arienzo
}

Clinica Ortopedica e Traumatologica del Policlinico dell'Università degli Studi di Palermo (Palermo, ITALY)

The aim of this paper is to carry out a retrospective clinical and radiological study on our patients treated in the Orthopaedic Clinic of Palermo University.

Between 2005 and 2008 we operated on 51 patients with fractures of the diaphysis of the humerus, 29 males and 22 females with a mean age of 53, the youngest being 19 and the oldest 93 . Forty fractures resulted from trauma, 11 were pathological of which 9 from metastatic tumours (1 from Paget and 2 from plasmocitoma).

In all patients the pin was blocked proximally with one or two screws and distally with a particular kind of anti-torsional blockage typical of the Seidal pins without screws.

Follow-up checks were carried out after 1, 3, 6 and 12 months. 46 patients were checked with an mean follow-up of 3 years (minimum 2-maximum 5). The fractures healed in 10-16 weeks; mobilization was generally early and the functional recovery was generally excellent. In the pathological fractures the "healing" time was generally longer. Of the 35 of the post-traumatic fractures 2 had neurological complications (lack of sensitivity of the radial nerve) but both recovered without any treatment. The results were excellent in 35 cases, fair in 7, poor in 4 .

In the pathological fractures we observed a downward movement of the pin in one case but it did not affect the elbow function. In all the cases there was an improvement in the pain.

The endomedullar pins have less morbidity. The Seidal pin has a single distal blockage with a screw that, when it withdraws proximally, allows the opening of three distal flanges that prevent torsional movements.

In the cases of pathological metastatic fractures, it is possible to carry out a palliative operation blocking the fracture site without touching it, eliminating the pain and improving the quality of life.

\section{Aseptic forearm nonunions treated by plate and fibular opposite and intercalary autograft}

C. Faldini, S. Pagkrati, M.T. Miscione, D. Leonetti, M. Nanni, F. Acri, S. Giannini

Istituto Ortopedico Rizzoli (Bologna, ITALY)

Objective Forearm nonunion frequently changes the relationship between the radius and ulna and may lead to impairment of forearm function. We propose a new surgical technique for aseptic forearm nonunions combining a fibular cortical autograft strut with a metal plate and a fibular intercalary autograft in cases with a segmental bone defect. Material and methods We retrospectively reviewed 20 patients with a mean age of 31 years (range, 17-48 years) at the time of surgery, presenting in 2 cases an isolated nonunion of the radius, in 14 cases an isolated nonunion of the ulna and in 4 cases the non-union of both bones. Nonunions were atrophic in 18 cases and ipertrophic in 6 cases. Minimum follow-up was 12 years (mean, 14 years; range, 12-21 years).

Results There were no intraoperative or postoperative complications. At last follow-up, all forearm bones had remodelled. The mean visual analog pain scale was 1 (range, 0-3). Forearm function improved; there were no radiographic signs of ankle arthritis at follow-up.
Conclusions Surgical treatment of aseptic forearm nonunions by combining a massive fibular cortical autograft strut with a plate and associating a fibular intercalary autograft in case of a segmental bone defect led to bone healing, improved forearm function, and a durable outcome with long-term follow-up.

\section{TRAUMATOLOGY 4}

\section{Treatment of the persubtrochanteric fractures of the femur with a new long pertrochanteric nail: preliminary results of 22 cases treated with long Veronail}

\author{
N. Godi, G. Peruzzi, F. Cortese, S. Rigotti, C. Zorzi
}

Ospedale Sacro Cuore, Negrar (Verona, ITALY)

Introduction The incidence of femoral persubtrochanteric fractures continues to rise. The scope of this study was to give a first evaluation of this new method of intramedullary osteosynthesis, Veronail (Orthofix, Verona) in its long configuration that provides fixation through two converging screws locked into the femoral head and a dynamic or static distal locking. This type of nail allows the surgeon to realize a stable synthesis at proximal and distal level, reducing therefore the post-operative pain, blood loss but especially it allows an early weight bearing upon the patient's operated leg with all the consequent benefits.

Material and methods From 1st January 2008 until 31st December 2009 twenty-two patients were treated with this new system in our Department: the fractures were classified according to the AO classification, all the fractures were treated with the long Veronail in its converging screws configuration locked into the femoral head and the distal locking configuration was decided on the basis of the fracture type. The functional recovery of the patients was assessed with the modified Harris Hip score.

Results All patients healed and did not experience any early or later complications, they have a good functional recovery. We believe that the use of this method for these fractures reduces intraoperative complications (surgery duration, blood loss, anesthesiologic time), improves patient's compliance during the postoperative period (immediate mobilization of the hip joint and knee joint, decrease of the hospitalization time) and the results (healing time, early weight bearing) in comparison with other methods.

Conclusions The exclusive configuration with the converging screws locked into the femoral head allows the surgeon to realize a stable osteosynthesis also in the persubtrochanteric and multifragmented fractures, and it represents a innovation among the intramedullary nails which are nowadays available in the market. This would be a preliminary study, and even if it is encouraging, due to the early results, more cases are required to properly evaluate the efficacy of this device.

\section{LCP trochanteric plate to treat comminuted proximal femoral fractures. Preliminary report}

\section{Lazzara, A. Aquino, F. Del Prete, G. Caruso, A. Petrini}

Ortopedia, Ospedale San Giovanni di Dio (Florence, ITALY)

From June 2009 at our Center we have introduced the LCP (Locking Compression Plate) trochanteric plate, which represents the latest 
evolution of the LCP system specifically designed for the treatment of unstable fractures of the trochanteric region, such as the A3 fractures (according to AO classification), with reverse or subtrochanteric extension, as a possible alternative to intramedullary nailing. It consists of a diaphyseal plate made of stainless steel with holes for the diaphyseal region (4-10) and a proximal part (shaped to fit to the trochanteric region, for this reason there are plates for left and right femur) where it is possible to apply 3 angle locking screws (two of 7.3 and one of 5) to secure the femoral neck reduction.

In our Department from June 2009 to March 2010 we performed 23 operations using this system. In particular, 17 women and 6 men aged between 64 and 96 years with a mean age of 82 years. Except for two cases: a pathological fracture and a fracture nonunion with breakage of the previously implanted DCS, the remaining 21 procedures were performed on recent fractures. We used a 4 holes plate in one case, 6 holes in 13 cases, 8 holes in 7 and a 10 holes plate in the remaining 2 cases (with the option to use locking or traditional diaphiseal cortex screws).

In these patients usually we do not grant an immediate post-operative weight bearing, but that decision was not taken on the basis of the means of synthesis, but on the type of fracture (mainly based on the severity of comminution).

All the patients had a clinical and radiographical follow-up every 40-45 days until consolidation occurred. The follow-up ranging from 9 months to 40 days. In some cases, those with more complex fracture rhymes, at the first control has been granted only partial load. All the fractures healed in a mean time of 3 months. We recorded only one complication, the shift of the proximal screws, probably due to a technical error, but the fracture was stable.

In our opinion, the preliminary results look encouraging, even allowing for the fact that we are limiting the use (as specifically noted) to those with particularly complex fractures where other methods of synthesis do not seem to give a tight enough.

\section{The percutaneous compression plate (PC.C.P) in the treatment of pertrochanteric fractures: functional recovery and mortality after 1 year and their correlation with the ASA classification}

\author{
R. Giancola $^{1}$, G. Antonini ${ }^{1}$, D. Berruti ${ }^{2}$, E. Blanchietti ${ }^{3}$, \\ V. Di Placido ${ }^{4}$, V. Francione ${ }^{5}$, P. Greco ${ }^{6}$, T.C. Russo ${ }^{7}$, \\ L. Pietrogrande ${ }^{8}$ \\ ${ }^{1}$ A.O. San Carlo Borromeo (Milan, ITALY); \\ ${ }^{2}$ A.O. San Gerardo (Monza-MI, ITALY); \\ ${ }^{3}$ Ospedale Civico (Chivasso-TO, ITALY); \\ ${ }^{4}$ Asur 2 (Urbino, ITALY); \\ ${ }^{5}$ A.O. Mazzini (Pesaro, ITALY); \\ ${ }^{6}$ A.O. Caserta (Caserta, ITALY); \\ ${ }^{7}$ A.O. Busacca Maggiore (Modica, ITALY); \\ ${ }^{8}$ A.O. San Paolo (Milan, ITALY)
}

Introduction Outcome of surgical treatment is superior to that of conservative treatment for hip fractures, nevertheless for some patients operation is unfit due to the poor and unstable medical conditions. Literature shows a higher prevalence of patients classified as ASA I or II in comparison with the ASA III and IV for surgical treatments. The ASA classification is a good predictor of mortality and recovery of the mobility, issue that remains a problem for patients with this kind of fracture.

Material and methods Here we present the results of 8 Italian Centers concerning 187 cases of $31 \mathrm{~A} 1$ and $31 \mathrm{~A} 2$ fractures treated with the percutaneous compression plate (PC.P.C) between March
2008 and January 2009. The parameters evaluated were the intraoperative details, mortality and function recovery (Harris hip score) at 1 year as well as their correlation with patient groups: ASA I-II (group A) and ASA III-IV (group B).

Results There were 138 women and 49 men with an average age of 82 years (18-104). $75 \%$ of patients was ASA 3 and 4 . The average duration of the operation was $43 \mathrm{~min}$. Postoperative blood transfusion was necessary in $49 \%$ of patients. The mortality at 1 year was $21 \%$ $(\mathrm{n}=39)$. The mortality between the groups was significantly different (group A: $4 \%$, group B: $25 \% ; p<0.05$ ). At 1 year the average modified Harris Hip Score was 56 points, $87 \%$ of pre-trauma value. The average change of the final score in comparison with the pretrauma value was not statistically significant (group A: -11 points; group B: -10 points; $p=0.146$ ).

Discussion The PC.C.P. fulfilled the concept of minimally invasive surgery and was efficacy used for the surgical treatment of fractures in fragile and very old patients. The ASA score has been confirmed as a predictor of mortality, but this plate permitted an equivalent functional recovery in both the groups.

\section{TRAUMATOLOGY 5}

\section{Treatment of the tibial plateau fracture with high energy by the hybrid external fixation}

\section{P. Barbato, F. Nicolosi, W. Leonardi}

Aornas Garibaldi, U. O. Ortopedia e Traumatologia, Presidio Garibaldi Nesima (Catania, ITALY)

Introduction The tibial plateau fractures are an important chapter of the trauma, in particulary those with high energy have a damage to the structures and to the function of the knee joint. As a matter of fact, because of the biomechanics of the anatomical components, a surgical treatment that maximally respects the anatomical zone is required.

Material and methods The patients subjected to surgical treatment were 18 until 70 years old with a peak between 30 and 50 years old. The fractures were evaluated with Shatzker classification, particulary the fractures of the III, IV, V and VI type. The Hybrid External Fixator was used as tool. This allowed the treatment of the bone damage without further harm of the soft tissue that covers the knee joint and the leg. After a trauma with high energy, the soft tissues are damaged and the surgery treatment often is delayed until the improvement of the soft tissue.

Results The use of that tool allows the treatment of the fractures reducing the damages to soft tissues and improve the rehabilitation of the knee joint. No patient showed infection of soft tissues and osteomyelitis. On average the patients had full loading after 45 days. The healing time was on average 150 days.

Conclusions The targets to be achieved in the treatment of these fractures are the anatomical reduction, the lasting synthesis and the improvement of rehabilitation. The hybrid external fixation proved itself as an efficient tool of synthesis for the achievement of these results.

\section{The mineral bone substitute in tibial plateau fractures}

\section{E. Betti, F. Gazzarri, L. Magistrelli}

Azienda Ospedaliera Universitaria Pisana (Pisa, ITALY)

Objective The treatment of the tibial plateau fractures is often hard because of the peculiarity of the articular damage. The serious 
collapse of the metaphyseal bone, located beneath the reconstructed articular surface, makes the osteosynthesis mechanically unsafe for long. Injecting and fast setting mineral bone substitute used to fill the bone void, with its initial mechanical strength allows to get optimal stability integrating minimal ostheosyntesis

Material and methods Percutaneus indirect articular reduction was performed by raising articular fragments wedged in the metaphiseal bone collapse. Temporarily fragments were held with a Kirshner wire and definitely fixed with a cannulated screw. Mineral bone substitute (Norian SRS) was injected to fill the residual bone void. Seventy patients affected by tibial plateau fractures (type B2 and B3 according to AO classification) were treated between 1997 and 2008 using this minimal invasive technique. Injectable and fast setting bone substitute Norian SRS, with early compressive strength, allows the mobilization the day after the surgery and partial weigh-bearing after 2 weeks. The minimum follow-up was 1 year. We used the Hohl assessment form for clinical evaluation, and the criteria of Rasmussen X-rays for the radiographs.

Results The final conclusions, resulting from integrated analysis of the clinical data and X-rays data, can be represented as follows: 62 cases excellent-good $(88.5 \%), 7$ fair $(10 \%)$, and 1 poor $(1.5 \%)$.

Discussion In these fractures, where the metaphiseal cancellous bone loss plays a leading role, the injectable and fast setting mineral bone substitute, with the complete filling of the metaphiseal bone void, provides an immediate mechanical stability that could not be otherwise safely ensured by any hardware.

Conclusions The treatment of unicondylar tibial plateau fractures (B2 and $\mathrm{B} 3 \mathrm{AO} / \mathrm{OTA}$ classification) with percutaneous indirect reduction, minimal ostheosynthesis and mineral bone substitute let us reach the goal of the immediate restoration of joint function and the prevention of late post-posthraumatic osteoarthritis.

Suggested readings

1. Gui L (1973) Fratture e lussazioni. Gaggi Ed., Bologna

2. Rasmussen PS (1973) Tibial condylar fractures. impairment of knee joint stabilitas an indication for surgical treatment. J Bone Joint Surg 55:1331-1350

3. Simpson D, Keating JF (2004) Outcome of tibial plateau fractures managed with calcium phosphate cement. Injury 35(9):913-918

\section{Applicability of the Solomin method to internal osteosynthesis} $\frac{\text { M. Massobrio, G. Antonietti, F. Necci, P. Albanese, C. Esposito, }}{\text { U. Bianco }}$

(Rome, ITALY)

Introduction There is no universal, objective, and scientific method of surgical description, concerning internal synthesis. The employment of osteosynthesis devices is not associated to an adequate description method. This complicates the study and the transmission of data for practical and research scopes. In 2002 Solomin [1] suggested the application of an unified method to describe osteosynthesis related to external fixation (MUDEF), in order to encode all the different application methods of this technique. We evaluated the possibility to use the descriptive alfa-number Solomin system in internal osteosynthesis.

Material and methods The Solomin method is a formula made by:

- Roman numbers from 0 to IX to show longitudinally the bone level where $k$-wire or the screw is inserted.

- Arab numbers from 1 to 12 to show, in a tomographic section, the sector of the circumference corresponding to the position of the $k$-wire or the screw.
- Comma (,) and hyphen (-) to distinguish the position and the orientation of the screw or $k$-wire or their route.

- Degrees showing the insertion angle.

Starting from 2007 we studied a group of patients $(n=40)$ affected by fractures and operated with internal osteosynthesis. We verified the applicability of the Solomin descriptive method to classify the performed treatment with this method. The method was applied to the 4 long segments: omerus, radius-ulna, femur, tibia-fibula. For each segment we randomly chose 10 patients affected by fractures of different types, classified with the A.O. method. They had been already treated with internal osteosynthesis. The method was applied on the post-operative X-rays, and we described the lesion location, the eventual exposition, the extension of the synthesis.

Results It was possible to apply the method in all cases, indicating precisely the fracture and exposition site, and the synthesis extension. The method does not seem to be useful in the description of osteosynthesis of the femoral proximal lateral and medial fractures.

Discussion and conclusions The system seems suitable to identify precisely the pathological levels, the osteosynthesis implant sites, and their extension. It is also effective to locate a neoplastic lesion or an altered morphologic bone lesion. Therefore it is applicable in radiotherapy, for example, for the identification and the mapping of the pathologic site. The system is compatible with the creation of a software [2] for the transmission and the storage of data. It could be possible the creation of a national archive of osteosynthesis.

\section{References}

1. Solomin L, Kornilov NV (2003) Importance of "Method of unified designation of external fixation". J Bone Joint Surg Br 86[Suppl 3]:300

2. Raguzzi PP, Mora R (2006) Future perspectives: computer-assisted surgery; Nonunion of the long bones. Springer-Verlag, Milan

\section{NEOPLASTIC DISEASES 1}

\section{A new scoring system to evaluate the risk of pathologic fractures in patients with bone metastases of the extremities}

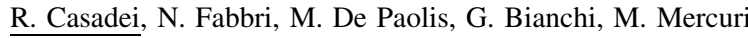

Istituto Ortopedico Rizzoli (Bologna, ITALY)

Objective We evaluated the effectiveness of Mirels score in bone metastases of the extremities.

Material and methods Three hundred and thirty-three metastases of the femur and 135 of the humerus were considered. Mirels system [1], applied to our patients, was not effective to predict pathologic fractures. Therefore, we evaluated many risk factors to find those statistically significant to elaborate a more objective scoring system. Results At a univariate statistical analysis only sex for the femur, age, site, isotype, longitudinal cortical erosion, circumferential cortical destruction, relative extension of the lesion were significant. However, at a multivariate statistical analysis, only age ( $<50$ years, 50-65 years, >65 years), primary tumor (good prognosis: breast, prostate, thyroid, kidney; fair prognosis: bowel, uterus, bladder; poor prognosis: lung, pancreas, stomach, melanoma, ovary), site (proximal, diaphyseal, distal), longitudinal cortical erosion (femur: $<3 \mathrm{~cm}$; 3-4 cm; $>4 \mathrm{~cm}$; humerus: $<2,5 \mathrm{~cm} ; 2.5-4 \mathrm{~cm} ;<4 \mathrm{~cm})$, circumferential cortical destruction $(<50 \%, 50-75 \%,>75 \%)$ remain significant factors. Appling a score to each factor according to the relative risk, we obtained a scoring system for the femur and one for the humerus. 
Age ( $<50$ years, $p=1 ; 50-65$ years, $p=2 ;>65$ years, $p=3$ ), isotype (good prognosis, $p=3$; fair prognosis, $p=2$; severe prognosis, $p=1$ ), femur (proximal, $p=3$; diaphyseal, $p=2$; distal, $p=1$ ), humerus (diaphyseal, $p=3$; proximal, $p=2$; distal, $p=1$ ), longitudinal cortical erosion (femur: $<3 \mathrm{~cm}, p=1 ; 3-4 \mathrm{~cm}, p=2$; $>4 \mathrm{~cm}, p=3$; humerus: $<2,5 \mathrm{~cm}, p=1 ; 2.5-4 \mathrm{~cm}, p=2 ;<4 \mathrm{~cm}$, $p=3)$, circumferential cortical destruction $(<50 \%, p=1 ; 50-75 \%$, $p=2 ;>75 \%, p=3)$. Patients with a score $>10$ were at high risk of pathologic fracture.

Discussion Mirels system is based on four factors: site, pain, extension, radiographic appearance and it is used in both lesions of femur and humerus, but the incidence of pathologic fractures is different in these sites. In fact, in the humerus pathologic fractures occur more frequently in the diaphysis whereas in the femur in the trochanter area. Moreover, Mirels considers pain as a significant factor, but it is a very subjective parameter, not statistically significant in our series. The radiographic appearance of the lesion is another not objective factor in Mirels system because radiographic evaluation is related to the quality of X-rays and the experience of the examiner.

Conclusions Our scoring system is more adequate because it is based only on objective factors and it is more accurate because it is different for femoral and humeral lesions. However, a more careful evaluation on a greater number of cases is required to validate this scoring system.

\section{Surgical treatment of the femur for metastases: an analysis of oncologic outcome and survival comparing nailing versus prosthetic replacement}

T. Calabrò, G. Drago, A. F. Mavrogenis, M. Romantini, G. Ussia, P. Ruggieri, M. Mercuri

Department of Orthopaedics, University of Bologna, Istituto Ortopedico Rizzoli (Bologna, ITALY)

Objective Femoral involvement is frequently observed in bone metastases, generally complicated by pathologic fractures. Aim of this study was to review surgical treatment of femoral metastases, comparing nailing versus resection and prosthetic reconstruction.

Material and methods Between 1975 and 2008, 110 patients were surgically treated for metastatic disease of the femur. Prostheses were implanted in 57 cases (16 HMRS Stryker, 38 MRP Bioimpianti, 2 Osteobridge and 1 GMRS). In 53 patients femoral nailing was performed with different types of locked nails (32 Gamma, 14 GrosseKempft and 6 T2-Stryker). Sites of primary tumor were breast (33 cases), kidney (18), lung (17), undifferentiated carcinoma (14), g.i. (8), bladder and prostate (5 each), endometrium and thyroid (3 each), skin (2), pheochromocytoma and pancreas (1 each). Indications to nails were given in patients with femoral metastasis and poor prognosis: multiple metastases, short free interval, unfavourable histotype, poor general conditions. Resection and prosthesis was preferred for patients with solitary metastasis, long free interval, favourable histotype, good general conditions or in whenever the extent of the lesion was not amenable to a durable internal fixation. Complications were analysed. Univariate analysis by Kaplan-Meier curves of implant and oncological survival was performed. Functional results were assessed with MSTS system.

Results Oncologic results showed: 23 patients alive with disease at a mean of 52 months, 57 patients died with disease at a mean of 9 months and 30 patients were lost to follow-up. Survival in patients treated with femoral nailing was about $10 \%$ at 5 years versus $20 \%$ for patients treated with resection. Patients with resections had a better survival curve at 2 years. Complications were: infections $(4 / 110$,
$3.7 \%)$, aseptic loosening (1/110, 1\%). No complications were observed in patients treated with nailing.

Conclusions Internal fixation is indicated in patients with multiple metastases and poor prognosis. When expected survival is longer and there is a solitary metastasis, resection is preferable. Indications of resections progressively increased due to newer medical treatment giving longer survival.

\section{Surgical treatment of cervical spine osteoblastomas}

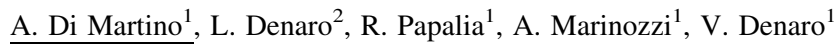 \\ ${ }^{1}$ Ortopedia e Traumatologia, Università Campus Bio-Medico (Rome, \\ ITALY); \\ ${ }^{2}$ Cattedra di Neurochirurgia, Università di Padova (Padua, ITALY)
}

Objective Main aim of the current work is to report the clinical and radiological picture and the results of surgery in nine patients affected by osteoblastomas at the cervical spine treated by a single surgeon with a standardized clinical and therapeutic approach.

Material and methods Nine patients affected by osteoblastomas at the cervical spine (Axial cervical spine, $\mathrm{n}=2$; subaxial, $\mathrm{n}=7 ; 6$ male and 3 female patients) were treated at our Institution in the last 8 years. Average age was 19 years, with a range of 7-24 years. Before surgery symptoms lasted on average 16 months (range 3-23 months). Diagnosis was confirmed by standard radiographs and bone scan, more often preformed before CT scan or MRI. CT guided biopsy was diagnostic for osteoblastoma in only 3 out of 9 patients. In all the other cases, the suspected diagnosis of osteoblastoma was confirmed postoperatively by pathological analysis. In all patients a marginal tumor resection with bone gap reconstruction with iliac crest harvested autologous bone graft, plus adjunctive fusion if required was performed. Preoperative arterography and tumor embolization was performed routinely in all patients.

Results Nocturnal neck pain responsive to Aspirin and NSAIDs was the common symptom. Only one patients presented with a C7 radiculopathy, while two patients showed symptoms related to spinal cord compression. Torticollitis was present in patients with upper cervical spine involvement, while a cervicothoracic scoliosis was a consistent finding in tumors arising a the lower cervical spine. At follow-up, an improvement of clinical and neurological findings was observed in all patients, without cases of local tumor relapse.

Discussion and conclusions Preoperative arterography and tumor embolization of hypervascularized lesions such as osteoblastoma was effective in our experience to decrease intraoperative blood loss and ease in finding a surgical cleavage during decompressive surgery.

\section{NEOPLASTIC DISEASES 2}

\section{GMRS prostheses in lower limb reconstructions in musculoskeletal oncology}

E. Pala, A. Angelini, T. Calabro', C. N. Abati, A. F. Mavrogenis, J. D. Valencia, P. Ruggieri, M. Mercuri

Department of Orthopaedics, University of Bologna, Istituto Ortopedico Rizzoli (Bologna, ITALY)

Objective To analyze results with a new modular uncemented tumor prosthesis for the lower limb (GMRS-Stryker) in the Rizzoli experience. 
Material and methods the GMRS Stryker Howmedica endoprosthesis (Stryker-Howmedica Inc, Rutherford, NJ) was used to reconstruct large segmental defects resulting from resection of the tumor. This newer model used is a modular system with a rotating hinge mechanism for the knee, uncemented straight-fluted stems, in titanium and chromium-cobalt-molybdenum with hydroxyapatite coating. Moreover adaptors are available to revise old implants HMRS Stryker with the new GMRS components (obtaining "Hybrid" implants). From October 2003 to December 2007, 175 GMRS prostheses were implanted, including 108 primary implants and 67 revisions after failures of 55 primary reconstructions after tumor resection and 12 implants for non oncologic reasons. Sites of reconstruction included: 118 distal femur, 35 proximal tibia, 21 proximal femur, 1 total femur. Histological diagnoses were: 102 osteosarcomas, 13 Ewing sarcomas, 13 chondrosarcomas, 12 other sarcomas, 3 metastases, 20 giant cell tumor. Retrospective analysis of complications and functional results (MSTS system) was performed and Kaplan-Meier curves of implant survival were defined.

Results At a mean oncologic follow-up of 2.5 years (min 2, max 5 years) 113 patients are continuously NED, 28 NED after treatment of relapse, $8 \mathrm{AWD}, 14 \mathrm{DWD}$. There were 13 major complications (7.4\%): 10 infections (5.7\%) at a mean time of 17 months and 3 aseptic loosening (1.7\%, at a mean time of 22 months from surgery: 2 in primary and 1 in secondary implants). Comparative statistical analysis of primary and secondary implants survival to major complications showed no significant difference. No cases of breakage were observed. Functional results evaluated in 154 cases were good or excellent in $96.7 \%$.

Conclusions Modular megaprosthesis replacement has become a universally accepted treatment option after bone tumors resection of the lower extremities. GMRS prosthesis is a good reconstructive option for large segmental defects in selected non-tumors conditions, such as complex revisions of prosthetic failures with massive bone loss and allograft non-unions or fractures. Middle term results with GMRS are promising with excellent functional results and low incidence of complications. Good implant survival needs to be confirmed at long term follow-up.

\section{Modular porous tantalum implants for reconstructive primary and revision tumor surgery}

\author{
N. Fabbri, P. Ruggieri, E. Rustemi, F. Vommaro, A. Angelini, \\ M. Mercuri
}

Istituto Ortopedico Rizzoli (Bologna, ITALY)

Objective Porous tantalum has been successfully used during the last decade for THA and TKA revision surgery associated with severe bone loss. Purpose of this study is to assess clinical results using porous tantalum implants for primary reconstruction after oncologic resection or revision surgery of a failed pre-existing tumor reconstruction.

Material and methods Retrospective study of 12 patients (6 male and 6 female) that underwent revision surgery of a failed tumor implant (9 cases) in the hip-pelvis ( 5 cases) or knee region ( 4 cases), and primary reconstruction of hip-pelvis after surgical resection of a malignant bone tumor. Mean age at the time of surgery was 35 years (22-55 years). Cause of failure in revision surgery was aseptic loosening (6 cases) or deep infection (3 cases). Revision of infected cases was performed in a staged fashion. All patients had a severe segmental bone defect as a result of either the treatment of primary tumor and/or the recent cause of failure. Bone defect was managed in all patients with modular porous tantalum implants, uncemented at the interface with host bone and cemented in the contact zone with augments, used in all cases, associated with morcellised bone grafts to fill residual cavitary defects and with a megaprosthesis in 7 cases (4 proximal femur, 2 distal femur, 1 proximal tibia). Minimum follow-up in all cases is longer than 2 years, mean follow-up is 3.8 years (2.5-6 years).

Results There was 1 recurrent infection requiring further surgical treatment. In all cases the tantalum implant is well fixed and functional at the most recent follow-up.

Conclusions Porous tantalum has been clinically very satisfactory at short term follow-up, demonstrating very reliable capability to obtain biologic fixation when a severe segmental bone defect is present. Longer follow-up is required to appreciate longer term shortcomings.

\section{Total femur megaprostheses for bone sarcomas: the Rizzoli experience}

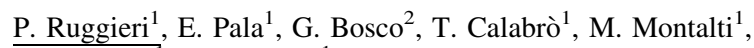
A. Angelini ${ }^{,}$M. Mercuri ${ }^{1}$

${ }^{1}$ Department of Orthopaedics, University of Bologna, Istituto Ortopedico Rizzoli (Bologna, ITALY);

${ }^{2}$ Department of Orthopaedics, Ospedale Maggiore (Bologna, ITALY)

Objective Aim of this study was to analyze the risk of local recurrence, survival and functional outcome in total femur resection and prosthetic reconstruction for bone tumors at the Rizzoli Institute.

Material and methods We retrospectively reviewed 23 patients with total femur megaprostheses implanted between 1987 and 2006 after resection of bone tumors; 22 patients had large sarcomas and one had metastatic osteosarcoma after previous distal femur resection. Two patients lost at follow-up were excluded; the remaining 21 included 15 male and six female with a mean age of 21 years. Istological diagnoses included: 12 osteosarcomas, 7 Ewing's sarcomas, 1 angiosarcoma, 1 fibrosarcoma. Oncologic results were evaluated and Kaplan-Meier survival curves were defined. Function was assessed according to the Musculoskeletal Tumor Society system.

Results At last follow-up, 7 of the 21 patients were continuously disease-free (CDF) at 148 months (all had preoperative chemotherapy), one patient had no evidence of disease (NED) after treatment of recurrence at 4.5 years and 13 patients dead with disease (DWD) at a mean time of 17 months (five osteosarcomas, six Ewing's sarcomas, one angiosarcoma, and one fibrosarcoma). Major complications included one vascular complication requiring hip disarticulation, one infection, one detachment of a trochanteric reattachment device for the glutei. One patient had polyethylene components changed at 53 months. Functional results in 15 evaluated patients were excellent (over $76 \%$ function) in 4 patients $(26.7 \%$ ), good (from 56 to $75 \%$ function) in 8 patients (53.3\%) and fair (from 26 to $50 \%$ function) in 3 (20\%). Average functional score was 20/30 (66\%). The overall continuously disease free survival of patients (patients surviving without evidence of recurrence or metastases) was $38 \%$ at 16 years. The continuously disease free survival (patients surviving without evidence of recurrence or metastases) of 11 patients with osteosarcoma was $45 \%$ at 10 years.

Conclusions Total femur prosthesis allows salvage procedure in tumors with extensive femoral involvement or in presence of skip lesion along the femur. The prognosis of these tumors is poor, but this reconstruction gives satisfactory function with a low rate of major complications. 


\section{NEOPLASTIC DISEASES 3}

\section{Primary bone lymphomas in adults}

G. Scoccianti ${ }^{1}$, D. A. Campanacci ${ }^{1}$, L. Rigacci ${ }^{2}$, B. Puccini ${ }^{2}$, M. Ippolito ${ }^{1}$, G. Beltrami ${ }^{1}$, R. Capanna ${ }^{1}$

${ }^{1}$ SOD Ortopedia Oncologica and ${ }^{2}$ SOD Ematologia, AOU Careggi (Florence, ITALY)

From 1999 to 2009 at the Hematology Department of the University of Firenze, in collaboration with the Orthopaedic Oncology Unit, 18 cases of primary bone lymphoma in adults were treated.

Most of the patients presented a histotype of diffuse large B cell lymphoma (15 cases; $83.3 \%$ ). In two patients a follicular lymphoma was detected; in one patient a small cell linfocitic lymphoma was diagnosed. Age of the patients ranged from 19 to 82 years (mean 53.7).

At presentation, 11 cases were classified as stage I and 7 as stage IV. In all cases no diffuse bone marrow involvement was detected by bone marrow biopsy. All skeleton sites were included in our series. 10 patients underwent a combination of chemotherapy and radiation therapy, 7 patients only chemotherapy, 1 patient only radiation therapy. Three patients received autologous stem cell transplant.

Only three patients underwent surgical treatment: one patient was affected by pathological fracture of the proximal humerus (resection and megaprosthesis), one patient by a pathological fracture of the tibia (fixation with plate), one patient by a vertebral fracture.

Five patients died of disease at a mean time of 12.2 months from diagnosis (range 3-36). One patient was lost at follow-up at 56 months from diagnosis and was at that time in disease remission. The remaining 12 cases are alive at a follow-up ranging from 4 to 125 months (mean 51.7). A complete remission was achieved in 12 cases; one of this patients presented a subsequent recurrence of disease. According to Kaplan-Meier analysis, survival at 5 years was $69.3 \%( \pm 0.11)$.

Prognosis of patients affected by primary bone lymphoma remains inconstant with cases presenting progression to death in a few months and cases with long survival, due to the efficacy of a combination treatment of chemotherapy and radiation therapy. Surgical treatment has few indications ( $16.7 \%$ of cases in our series underwent surgery) and should be limited to the patients affected by pathological fractures.

\section{Tumors of the hand and wrist}

A. Angelini ${ }^{1}$, M. Montalti ${ }^{1}$, G. Guerra ${ }^{1}$, E. Pala ${ }^{1}$, G. Ussia ${ }^{1}$, E. Rimondi ${ }^{2}$, M. Alberghini ${ }^{3}$, P. Ruggieri ${ }^{1}$

${ }^{1}$ Department of Orthopaedics, University of Bologna, Istituto Ortopedico Rizzoli (Bologna, ITALY);

${ }^{2}$ Department of Radiology and ${ }^{3}$ Department of Pathology, Istituto Ortopedico Rizzoli (Bologna, ITALY)

Objective Tumors of the hand and wrist are rare. Although most of these are benign, a failure to appreciate their presence may delay diagnosis and treatment. A thorough understanding of the differential diagnosis and a comprehensive strategy for evaluation are central for effective treatment. Aim of this study was to evaluate the incidence, histologic features, treatment strategy of the most common tumors involving hand and wrist.

Material and methods Between 1900 and 2007, 1,491 tumors of the hand or the wrist were retrospectively analyzed. Imaging included radiographs in all patients, and CT or MRI when feasible. Diagnosis was established in all cases with biopsy and histologic slides were reviewed. The lesions arose from soft tissue in 12 cases, and from bone in the remaining cases. Benign or pseudotumoral lesions were 1,372 (92\%): multiple chondromas (499), enchondroma (362), giant cell tumor (141), osteoid osteoma (71), solitary osteochondroma (57), "brown tumors" in primary hyperparathyroidism (54), aneurysmal bone cyst (50), reparative giant cell granuloma (26), periosteal chondroma (21), and other entities with less than 15 cases each. Malignant lesions were 119 (8\%): metastatic carcinoma (28), osteosarcoma (29), chondrosarcoma (26), Ewing's sarcoma (17), and other more rare entities. Localizations were phalanges $(756 ; 50 \%)$, metacarpal region $(369 ; 25 \%)$, and the wrist $(366 ; 25 \%)$.

Results Benign lesions were generally treated with curettage \pm bone grafting. Giant cell tumors recurred frequently after curettage. For malignant lesions, chemotherapy is required pre- and postoperatively, and especially when the tumor involved the phalanges, partial or total amputation may be required.

Conclusions Malignant tumors are relatively rare, but we re-emphasize the need to maintain a high level of attention on imaging, even when diagnosis seems straightforward. Whenever there is a doubt on the lesion, a biopsy should be done before proceeding with the definitive surgical procedure. In spite of the complex anatomy, adherence to proper oncologic principles most often will lead to a satisfactory outcome.

\section{Bone metastases of the foot: report of three cases}

\author{
M. Marinelli, N. Orlando, C. Chillemi, G. Zanoli, L. de Palma
}

Cattedra di Ortopedia e Traumatologia, Università Politecnica delle Marche, Azienda Ospedaliero-Universitaria Ospedali R (Ancona, ITALY)

Bone metastases of the foot are rare and often misdiagnosed. Only tarsal localizations are relatively frequent. Primary tumors are mostly adenocarcinomas (colon, kidneys, lungs). Three cases of acrometastases, localized, respectively, on the ungul phalanx of the hallux, the first metatarsal and the first cuneiform, are reported. Primitive tumor sites differed in the three case (renal, pulmonary and mammary origin). Adequate therapy must be preceded by an accurate staging. Surgery should aim to excise the tumor and restore, when possible, a good function of the foot without pain. Amputation should only be resorted to if it is not possible to perform the excision of the tumor in patients whose life expectancy is not too short. Wide local and systemic diffusion of the tumor and a short life expectancy are contraindications for any surgical approach. Treatment, in these cases, must try to achieve a satisfactory pain control and decrease the local expansion of the tumor.

\section{INFECTIOUS DISEASES 1}

\section{Role of frozen section histology for diagnosis of infection in hip and knee arthroplasty revision surgery}

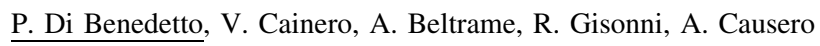

Clinica Ortopedica, Azienda Ospedaliero-Universitaria (Udine, ITALY)

Background The diagnosis of a suspected infected prosthesis is often difficult, although it is important for the choice of treatment. Even at 
surgery, it is not easy to assess whether the prosthesis is infected or not even though this may be important for the choice of surgical procedure. Intraoperative frozen section analysis is used in evaluating possible infections in cases of hip and knee revision arthroplasty, serving as an adjunct to preoperative and intraoperative studies [1]. Patients and methods Between November 2008 and January 2010 we examined sections and cultured periprosthetic tissues in prosthetic revision in 41 cases, including 23 hips and 18 knees. For preoperative diagnosis, we used the history (pain, drainage), laboratory analysis [erythrocyte sedimentation rate (ESR), protein C, blood count], radiology, nuclear medicine and synovial fluid analysis. Nevertheless these tests showed low confidence levels [2]. We assessed sensitivity (SE), specificity (SP), positive predictive value (PPV), negative predictive value (NPV). Samples of tissues were taken to be analyzed immediately from frozen sections, to be processed on a routine basis later, and to be referred for bacteriological cultures. A finding of 5 or more polymorphonuclear leukocytes per field at a magnification of $400 \times$ was considered positive for infection.

Results The analysis of frozen sections for infections was compared with the results of routine cultures. Concordance index between frozen sections and cultures was $86.9 \%$ and the Chi-Square Test was not significative. Comparison with the results of cultures showed a sensitivity of $71.4 \%$, a specificity of $93.7 \%$, a PPV of $83.3 \%$, an NPV of $88.2 \%$.

Conclusions In hip and knee prosthetic revision surgery frozen section analysis has a good specificity and sensibility, but the absence of polymorphonuclear leukocytes does not exclude periprosthetic infections. It is a quick and inexpensive test and we believe that frozen sections are of value in revision surgery when infections cannot be ruled out.

\section{References}

1. Kanner WA, Saleh KJ, Frierson HF Jr (2008) Reassessment of the usefulness of frozen section analysis for hip and knee joint revisions. Am J Clin Pathol 130(3):363-368

2. Levitsky KA, Hozack WJ, Balderston RA, Rothman RH, Gluckman SJ, Maslack MM, Booth RE Jr (1991) Evaluation of the painful prosthetic joint. Relative value of bone scan, sedimentation rate, and joint aspiration. J Arthroplasty 6(3):237-244

\section{Two-stage reimplantation for infected total knee arthroplasty: diagnostic challenges and timing of reimplantation}

M. Lo Presti, F. Iacono, D. Bruni, A. Bondi, M. P. Neri, G. Raspugli, M. Marcacci

\section{Istituto Ortopedico Rizzoli (Bologna, ITALY)}

Diagnosis and treatment of infection after TKA is very demanding because there is no single test with proven absolute accuracy for the diagnosis of PJI. The purpose of this study was to evaluate the ability of two-stage procedure to eradicate infection and to determine the timing of reimplantation after resection arthroplasty. Our cohort consisted in 87 patients operated between January 1998 and December 2006. In all cases the Total Condylar version III (TCIII) was implanted. The most frequently isolated bacteria was the Staphylococcus epidermidis (23\%), followed by Staphylococcus Aureus ( $8 \%$ ). At the present time, diagnosis remains dependent on serologic tests, analysis of aspirated joint fluid, 99mTc-labeled granulocyte scintigraphy and interpretation of intraoperative tissue and fluid results. Our data show that two stage exchange arthroplasty controlled infection in 77 out of 87 patients (89\%). Serologic tests (ESR, CRP, Plasma Fibrinogen) may be used as screening exams,

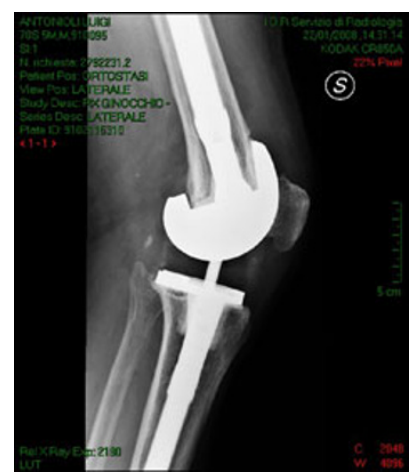

Fig. 1

while intraoperative tissue biopsy allows us to individuate microorganism in $58 \%$ of cases. At the same time granulocyte scintigraphy may be useful before reimplantation because of its high negative predictive value

\section{Primary arthroplasty with antibiotic loaded spacer in septic arthritis as first step of hip replacement}

\section{Portigliatti Barbos, S. Marino, A. Barberis, B. Mognetti}

Department of Clinical and Biological Sciences, University of Turin (Turin, ITALY)

The use of interim antibiotic loaded prosthesis-spacer in the treatment of infected hip arthroplasty has become a wide spread strategy in orthopaedic surgery. However there is poor experience of primary arthroplasty with antibiotic loaded spacer in septic arthritis as first step of definitive hip replacement. The benefits of this method are: direct treatment of source of infection and maintenance of joint mobility avoiding tissue contraction.

In a 57 y.o. woman contracting septicemia following lymphnode exeresis, S. aureus was isolated and antibiotic therapy adopted. Despite arthrocenthesis and drainage (under TC control) evidence of septic necrosis of the left hip drove to open debridement. In the same surgical time we implanted an antibiotated prosthesis spacer with the aim to save length of limbs and articularity waiting sepsis healing. Five months later spacer was removed and definitive arthroplasty was performed.

Observations: two luxations of implant (described complication) were observed in post-op of antibiotated spacer, probably due to muscle atrophy and non perfect congruence of prostheses spacer (only three dimensions of spacer/G Tecres are available).

Final result of arthroplasty was very good at 15 months after removing spacer and implant of total hip prosthesis.

In conclusion, the use of antibiotated hip prosthesis spacer is very uncommon as primary implant because of risk of instability and surgery difficulties of reimplant, but in our experience it was an effective method for primary treatment of severe hip infections as first step of definitive arthroplasty, avoiding scar formation, leg shortening, osteoporosis from disuse, distorted anatomy with loss of articularity, and permitting identification of infecting organism, determination of antibiotic sensitivity, appropriate adjustment of antibiotic therapy and local release of antibiotic from antibiotated prosthesis spacer. 


\section{Surface oxidation of prosthetic UHMWPE and bacterial adhesion}

E. Brach Del Prever ${ }^{1}$, A. Bistolfi ${ }^{1}$, M. Boffano ${ }^{1}$, P. Bracco ${ }^{2}$, L. Costa ${ }^{2}$, G. Banche ${ }^{3}$, V. Allizond ${ }^{3}$, A. M. Cuffini ${ }^{3}$

${ }^{1}$ Dipartimento di Traumatologia, Ortopedia e Medicina del Lavoro, AO CTO/Maria Adelaide, Università degli Studi di Torino (Turin, ITALY);

${ }^{2}$ Dipartimento di Chimica IFM, Università degli Studi di Torino (Turin, ITALY);

${ }^{3}$ Dipartimento di Sanità Pubblica e Microbiologia, Università degli Studi di Torino (Turin, ITALY)

Objective Peri-prosthetic infections are rare but, when they happen, imply very invasive and high-costly treatments. Some bacteria produce a biofilm over a biomaterial surface. The biofilm strongly adheres to the surface and obstructs antibiotics and umoral immunity intervention. The key-point is the initial bacterial adhesion. In this study we hypothesized that different kinds of Ultra High Molecular Weight Polyethylene (UHMWPE) with different surface properties could modify bacterial adhesive behaviour. The adhesion of biofilm-producing bacteria on standard UHMWPE and vitamin E-added UHMWPE was studied.

Material and methods Cylinders of standard UHMWPE and UHMWPE GUR 1050 annealed with vitamin E 0.5 and $0.1 \%$ were prepared. Biofilm-producing bacterial strains of Staphylococcus epidermidis ATCC 35984 were cultured on Tryptic Soy Agar (Merck KGaA, Darmstadt, Germany). Bacterial suspension was inoculated in tubes with cylindric samples. After centrifugation, bacterial adhesion was assayed at different incubation times $(3,7,24 \mathrm{~h})$. The results were analyzed by descriptive statistics and tested by unpaired TStudent test, in order to highlight significant differences $(p<0.05)$ between the various biomaterials.

Results At 3 and $7 \mathrm{~h}$ of in vitro incubation no significant differences between the two materials could be observed. On the contrary, at $24 \mathrm{~h}$ the bacterial adhesion on the vitamin E charged UHMWPE ( 0.1 and $0.5 \%$ ) was significantly lower than on standard UHMWPE. No difference was detected between the samples with the two different concentrations of vitamin E.

Discussion and conclusions The results suggest that the bacterial adhesion is lower on UHMWPE + vitamin E, probably due to the antioxidative properties of vitamin E. They also suggest that a minimal amount of the antioxidant is effective in reducing the bacterial adhesion. Further studies are needed to investigate the possible role of the oxidation and of the surface properties in bacterial adhesion. These studies can have relevant clinical implications and can contribute to the study and the comprehension of prosthetic infections. If a reduced bacterial adhesion is demonstrated for the vitamin $\mathrm{E}$ charged UHMWPE, this can make the material more attractive for a potentially higher resistance to infections.

\section{Disposable Antibiotic Coating (DAC) for infection prophylaxis of orthopaedic implants. An experimental study}

\section{$\underline{\text { C. Romanò }}{ }^{1}$, E. Meani ${ }^{2}$, R. Giardino ${ }^{3}$, G. Giammona ${ }^{4}$}

${ }^{1}$ Istituto Ortopedico I.R.C.C.S. Galeazzi (Milan, ITALY);

${ }^{2}$ Istituto Ortopedico G. Pini (Milan, ITALY);

${ }^{3}$ Istituto Ortopedico I.R.C.C.S. Rizzoli (Bologna, ITALY);

${ }^{4}$ Università di Palermo (Palermo, ITALY)
Objective Various antibiotic coatings have been proposed to prevent bacteria colonization and infection of orthopaedic implants. While most of the available technologies seem to provide an effective implant protection from infection, unknown long-term effects of antibiotic coatings raise some concerns for extensive application. Aim of the present study was to develop and test a new fast-resorbable antibacterial carrier (Disposable Antibiotic Coating, DAC), to be used as a temporary coating to prevent early bacteria colonization of metallic implants.

Material and methods The patented tested hydrogel (Fig. 1) is a copolimer comprising hyaluronic acid (HA) and a biocompatible polyester (poly-lactic acid) with or without polyethylene glycol chains to further modulate hydrophilicity and anti-fouling characteristics of the compound. The HA derivative is then added to water and mixed, just before its use, with the chosen antibacterial agent. For the purpose of this study, different HA-PLA derivatives have been tested, with two vancomycin and tobramycin concentrations and manually spread to uniformly cover the surface of a titanium specimen. To evaluate the release of vancomycin or tobramycin, high performance chromatographic analysis (HPLC) was carried out.

Results Antibacterial hydrogels provided vancomycin release ranging from 47 to $80 \%$ in 2 hours to $100 \%$ (complete release) in $24-72 \mathrm{~h}$, with antibiotic concentrations up to 400 times the minimum inhibiting concentration. The combined release of the two antibiotics (1\% w/v) showed $26.8 \%$ release of vancomycin and $35.8 \%$ of tobramycin at $2 \mathrm{~h}$ and complete release at $72 \mathrm{~h}$. Doubling antibiotic concentration $(2 \%$ w/v), yielded $56.6 \%$ and $76.6 \%$ antibiotic release, respectively for vancomycin and tobramycin at $2 \mathrm{~h}$ and complete release at $48 \mathrm{~h}$.

Discussion The Disposable Antibiotic Coating (DAC) has been showed in this study to completely release in vitro different antibiotics in $48-72 \mathrm{~h}$ at high local concentrations.

Conclusions HA chemical derivatization with polyesters leads to the formation of copolymers which can be used to produce antibacterial hydrogels with promising applications in the orthopedic field. These antibacterial hydrogels are in fact easily prepared and spread over a surface, showing the ability of releasing high concentrations of antibiotics for a desired, limited, period of time. Adding antibiotics to the hydrogel just before its use, allows customized antibiotic choice and dosing, avoiding shelf-life problems.

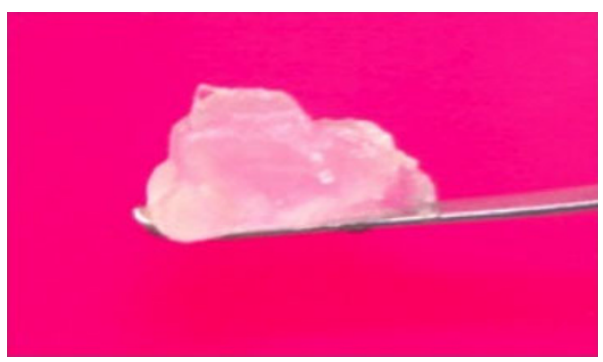

Fig. 1

\section{INFECTIOUS DISEASES 2}

\section{Pott's diseases: medical and surgical treatment in different type of spinal tuberculosis}

C. Zorzi, S. Rigotti, C.A. Luzi, N. Godi, A. Campacci, P. Avanzi

Ospedale Sacro Cuore, Negrar (Verona, ITALY) 
Objective The aim of the study is to integrate with minimal invasive treatment the surgical approach of spinal tuberculosis following Oguz's criteria [1].

Material and methods Between November 2009 and February 2010 we treated 3 patients with spinal tuberculosis, two women and one man, age $34(26 \pm 41)$ with 2 dorsal and 1 dorso-lumbar location; nobody presented neurological deficit. We have studied patients with X-ray, CT, MRI with contrast, biopsy and classified them into three different types IA/B, II and III according to Oguz. Medical therapy was used with Ethambutol $(15 \mathrm{mg} / \mathrm{kg}$ ), Isoniazid $(5 \mathrm{mg} / \mathrm{kg})$, Pyrazinamide $(25 \mathrm{mg} / \mathrm{kg})$ and Rifampicin $(10 \mathrm{mg} / \mathrm{kg})$ according to Bhojraj [2]. Indication for surgery was based on McLain's criteria [3] and we used Oguz's guide for surgical treatment: 2 patients of type IB were treated with drugs and abscess drainage; one of them became type II and was subjected to percutaneous posterior spinal stabilization; one type III was treated with drugs and standard posterior internal fixation.

Results Three months after abscess drainage and medical therapy with bracing, type IB patients were evaluated with CT. One patient with dorsal location showed an abscess resolution. One patient with dorso-lumbar location, had a cold abscess and destruction of bone and disc material with worsening of sagital index despite bed rest and bracing. We had treated this patient with posterior percutaneous stabilization. After 3 months CT evaluation showed normal sagital index and abscess solution. One type III patient with dorsal location was evaluated with CT, 3 months after posterior internal fixation and medical therapy, and showed bone graft, correction deformity and no abscess.

Discussion We agree with Oguz classification guide for surgical treatment, although it doesn't consider the anatomic localization considered by Bhojraj. The different locations between dorsal, dorsolumbar and lumbar need a different criterion for surgical approach.

Conclusions Type II patients with pathological fracture, kyphosis and stable deformity without neurological deficit can be treated with miniinvasive posterior percutaneous stabilization, instead of more invasive anterior debridement and fusion.

References

1. Oguz E, Sehirlioglu A, Altinmakas M, Ozturk C, Komurcu M, Solakoglu C, Vaccaro AR (2008) A new classification and guide surgical treatment of spinal tuberculosis: original paper. Int Orthopaed (SICOT) 32:127-133

2. Bhojraj S, Nene A (2002) Lumbar and lumbosacral tuberculous spondylodiscitis in adults: Redefining the indications for surgery. JBJS [Br] 84-B:530-534

3. McLain R, Isada C (2004) Spinal tuberculosis deserves a place on radar screen: review. Cleveland Clinic Journal of Medicine 71(7):537-548

\section{ARTHROSCOPY 1}

\section{Does the nanohydroxyapatite-based bone graft substitute improve bone-graft integration after ACL reconstruction with hamstrings? An MRI study}

A. Vadalà, R. Iorio, F. Conteduca, L. Basiglini, J. Conteduca,
G. Argento, A. Ferretti

Orthopaedic and Traumatology Department, University La Sapienza (Rome, ITALY)

Objective Several experimental and in vivo studies have shown that there is a tendon-bone fixation around 6-12 weeks after ACL reconstructions with hamstrings. The purpose of our study is to evaluate the effectiveness of the use of nanohydroxyapatite on improving bone-tendon integration after ACL reconstruction with hamstring tendons.

Material and methods Twenty consecutive male patients operated for ACL reconstruction were enrolled in this prospective study and they were randomly assigned to group A (10 patients, nanohydroxiapatite group) and group B (10 patients, control group). All patients underwent an MRI exam at 30,90 and 180 days after surgery with the aim to evaluate the following parameters as signs of bone-graft integration: (1) bone edema; (2) signal intensity in the central, in the peripheral part of the graft, and around the bone tunnel; (3) interface bone-graft; (4) amount of tunnel enlargement.

Results Clinical evaluation did not show any difference between the two groups. Radiologically, regarding bone edema, in both groups edema tended to decrease gradually and then disappear by the 180day follow-up. Regarding signal strength, at 180 days in both groups there was a recovery of basic hypointensity from the periphery toward the center. Regarding graft-bone interface, at 90 days, in Group A the interface thickness was $0.5 \mathrm{~mm}(0.4-0.8)$, while in Group B it was 0.8 (0.7-1.1); at 180 days, no hyperintensity bands were detected in any patients of both groups. Mean tibial tunnel diameter increased significantly from $9.04 \pm 0.06 \mathrm{~mm}$ to $9.68 \pm 0.13 \mathrm{~mm}$ in group A and from $9.03 \pm 0.26 \mathrm{~mm}$ to $10 \pm 0.43 \mathrm{~mm}$ in group $\mathrm{B}(p<0.05)$. No differences were detected on the femoral side $(p>0.05)$.

Conclusions Our study demonstrates that patients treated with nanohydroxyapatite show improved results in terms of resolution of cancellous bone edema, graft signal intensity, tendon-bone interface gap, and bone tunnel widening; moreover the use of nanohydroxyapatite did not expose patients of group A to higher incidence of any type of complications compared to patients of group B.

\section{The treatment by shock waves of the stiff knee after anterior cruciate ligament surgery}

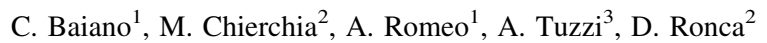

${ }^{1}$ Physical Medicine and Rehabilitation Division, Hospital A. Rizza, ASP (Syracuse, ITALY);

${ }^{2}$ Department of Orthopaedics and Traumatology, 2nd University (Naples, ITALY);

${ }^{3}$ Recovery and Rehabilitation Division of Campiglia Marittima, ASL 6 (Livorno, ITALY)

Objective The arthrofibrosis after ACL surgery is due to a formation of reactive connective tissue in and around the joint which can lead to fibrosis and ossification of the periarticular structures. Shelbourne defined arthrofibrosis any symptomatic reduction of joint movement and classified the joint stiffness into four stages according to severity of the process. Several authors suggest a surgical treatment. The option of using extracorporeal shock waves (ESWT) arises from therapeutic success obtained by this method in various calcified degenerative diseases of capsular, ligament and tendon structure.

Material and methods In an outpatient setting, 20 patients with stiff knee after ACL surgery (average time after surgery: 6-8 months) were randomly divided into two groups. The first group was treated with ESWT, (by Medical Space Orthospec electrohydraulic lithotripter). Each patient underwent a treatment of one session by week for 4 weeks, (approximately 2,500 shocks for session, with increasing energy intensity from 0.18 to $0.28 \mathrm{~mJ} / \mathrm{mm}^{2}$ ). The second group underwent a conventional physical treatment (ultrasound, TENS and laser therapy, five sessions by week for 4 weeks). Both groups underwent a kinesitherapy program to strengthen and to balance knee muscles. The 
evaluation was performed at the beginning of the treatment, at the end of the treatment and 2 months after the end of the treatment. We evaluated subjective pain during motion (VAS), joint range of motion (ROM) and disability (FIM). Analysis of results was performed in two ways: (1) intra group, with the Wilcoxon test; (2) inter group with the MannWhitney test, with significance of $p<0.05$.

Results The results indicate that in the treatment of knee stiff after ACL surgery, the association ESWT-kinesitherapy has a greater effect on reducing pain and restoring the range of motion than others therapy.

Discussion Many factors can cause stiffness of the knee after ACL surgery. Several authors reported an increased incidence of arthrofibrosis following open surgery and in patients operated too early after the trauma. The loss of the extension is the most important joint damage which reduces notably the daily activities. In this study, we considered particularly the restoring of extension by combining ESWT and kinesitherapy.

Conclusions There are numerous limitations in our study: small number of patients, different operators, different sort of transplant in ACL surgery, but in our experience the use of ESWT represented a valid therapeutic option in a conservative rehabilitation program to supplement kinesitherapy.

\section{ARTHROSCOPY 2}

\section{Does chronic MCL laxity in the setting of $\mathrm{ACL}$ reconstruction influence the clinical results? Prospective evaluation from surgery at a minimum 3-year follow-up}

S. Zaffagnini, T. Bonanzinga, G.M. Marcheggiani Muccioli, S. Bignozzi, G. Giordano, M. Nitri, N. Lopomo, M. Marcacci

Laboratorio di Biomeccanica, Istituto Ortopedico Rizzoli (Bologna, ITALY)

Objective Combined ACL+MCL lesions are challenging to treat. A time zero evaluation with a navigation system showed that patients with preoperative Anteromedial (AM) instability had similar Anteroposterior (AP) laxity at $30^{\circ}$ but greater valgus laxity at $30^{\circ}$ after the ACL reconstruction. This study investigates the same cohorts of patients at 3 years follow-up to see if the residual valgus laxity led to different results.

Material and methods In the previous study 57 patients were included, 20 with ACL+MCL lesion (group AM) and 37 with pure ACL lesions (group A). All patients underwent arthroscopic double- bundle ACL reconstruction with STG. In group AM the MCL lesion was treated non-operatively. Fifty-one patients were available at follow-up: 19 for group AM and 32 for group A. AP laxity was measured using KT-2000 with Manual Maximum Test at $30^{\circ}$; valgus laxity was evaluated with a Telos X-Ray test with a $10 \mathrm{Nm}$ valgus force. We evaluated clinical scores (IKDC, Lysholm, Tegner and Womac), muscle girth and time to return to activities.

Results We did not find any statistically significant difference concerning to clinical evaluation. A statistically significant difference was found in the medial joint opening during the valgus stress radiographs on the reconstructed knee $(1.7 \pm 0.9 \mathrm{~mm}$ group AM, $0.9 \pm 0.7 \mathrm{~mm}$ group $\mathrm{A} ; p=0.013$ ). Finally there was no statistically significant difference respect to the AP laxity at $30^{\circ}$ considering neither the AP displacement on the index side $(5.8 \pm 1.9 \mathrm{~mm}$ group $\mathrm{AM}, 5.3 \pm 2.4 \mathrm{~mm}$ group A) or the side to side difference $(2.4 \pm 2.5 \mathrm{~mm}$ group AM, $1.3 \pm 2.2 \mathrm{~mm}$ group A) (Fig. 1).

Conclusions The results showed that the residual valgus laxity found at time zero in the group AM still exist but it did not affect the AP laxity significantly at a minimum follow up of 3 years. A trend was seen for AM group to have higher AP laxity value but this did not affect functional results. These findings suggest that no additional surgical procedure on the MCL lesion is needed in combined lesions.

\section{Intra-operative analysis of knee dynamic stability during ACL reconstruction: anatomic double-bundle versus single-bundle plus lateral plasty}

\section{S. Zaffagnini ${ }^{1}$, N. Lopomo ${ }^{1}$, S. Bignozzi ${ }^{1}$, T. Bonanzinga ${ }^{1}$, C. Signorelli ${ }^{\mathrm{T}, 2}, \mathrm{M}$. Marcacci ${ }^{1}$}

${ }^{1}$ Istituto Ortopedico Rizzoli (Bologna, ITALY);

${ }^{2}$ Politecnico di Milano (Milan, ITALY)

Introduction Pivot-shift test is commonly used to qualitative assess the dynamic stability of the knee. Different ACL reconstructions are available: with a rationale, a specific reconstruction wanted to combine intra and extra-articular procedures using a single-blundle (SB) with an additional extra-articular lateral plasty-LP; with a different rationale the anatomical double-bundle (ADB) reconstruction was proposed trying to mimic the native ACL. The objective of this study was to quantify intra-operatively the recovery given both by an ADB reconstruction and by a SB+LP one, by means of PS test.

Material and methods 15 patients that underwent ADB ACL reconstruction and 10 patients that underwent SB+LP ACL reconstruction were involved in the study. A commercial navigation system (Orthokey, USA) was used for kinematic acquisitions. The surgeon
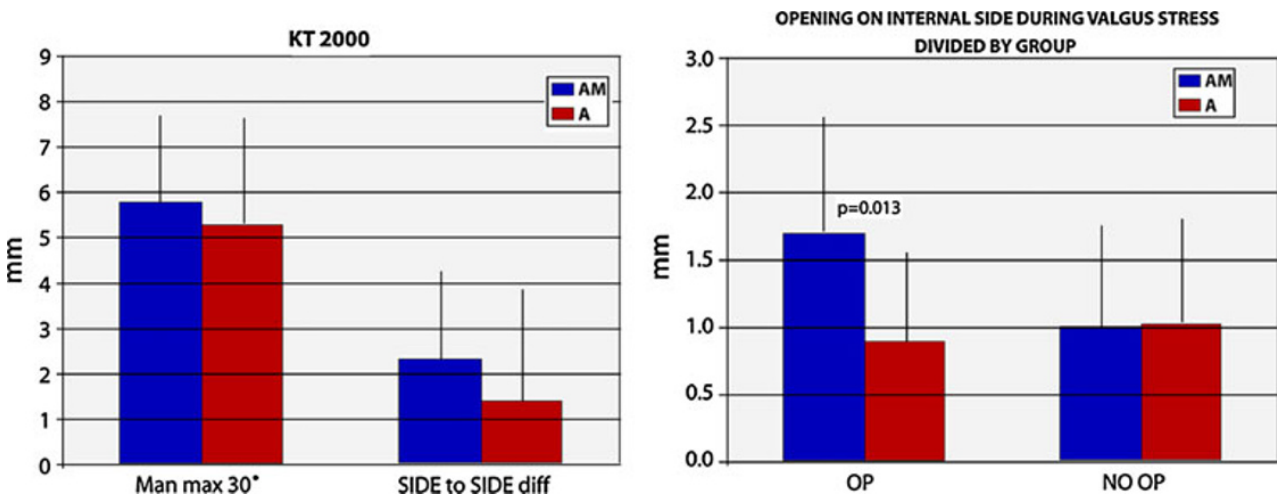

Fig. 1 
performed PS test before and after ACL reconstruction. We analysed the maximal anterior translation of the tibial lateral compartment during subluxation and the acceleration reached by the lateral compartment during tibial reduction.

Results All the dynamic instabilities (translation, acceleration) were significantly reduced both by the $\mathrm{ADB}$ and $\mathrm{SB}+\mathrm{LP}$ reconstruction $(p<0.0005)$. There were not statistical significance if we considered the recovery in anterior translation due to both the techniques $(9.5 \pm 7.7 \mathrm{~mm}$ and $10.3 \pm 2.1 \mathrm{~mm}$ respectively, $p=0.7656)$. SB+LP patients presented more acceleration after the reconstruction $\left(457 \pm 110 \mathrm{~mm} / \mathrm{s}^{2}\right.$ versus $\left.661 \pm 208 \mathrm{~mm} / \mathrm{s}^{2}, p=0.0060\right)$, but there were no statistical differences in the recovery due to both the reconstructions ( $p=0.420$ ). Conclusions The presented intra-operative analysis of knee stability allowed to highlight possible different dynamic behaviours due to the use of two different ACL surgical reconstructions. All ACL-deficient knees showed positive pivot-shift test before the surgery revealing huge values in the tibial subluxation and in the acceleration reached by the tibia, that is linked to tibial reduction during PS phenomenon. However, even with some difference due to the execution of the test, both $\mathrm{ADB}$ and $\mathrm{SB}+\mathrm{LP}$ reconstruction seemed to well control pivotshift phenomenon reducing tibial translation and thus controlling the dynamic instability of the joint.

\section{Results of isolated postero-lateral corner reconstruction}

L. Camarda $^{1}$, V. Condello ${ }^{2}$, V. Madonna ${ }^{2}$, F. Cortese ${ }^{2}$, G. Caradonna ${ }^{1}$, M. D'arienzo ${ }^{1}$, C. Zorzi ${ }^{2}$

${ }^{1}$ Department of Orthopaedic and Traumatology, University of Palermo (Palermo, ITALY);

${ }^{2}$ Department of Knee Surgery, Ospedale Sacro Cuore Don Calabria di Negrar (Verona, ITALY)

Background Isolated postero-lateral corner (PLC) tears are relatively rare events. Various surgical techniques to treat postero-lateral knee instability have been described; because surgical results are linked to cruciate reconstructions it has been difficult to date to define whether one surgical procedure has better prognosis than another. The goal of this study is to determine the clinical outcome of PLC reconstruction following fibular-based technique.

Material and methods We retrospectively evaluated a case series of patients who received isolated PLC reconstruction between March 2005 and January 2007. Ten patients were surgically treated for isolated injuries and were available for follow-up; mean patient age was 27.4 years (range 16-47 years). All patients were treated following the fibular-based technique: double femoral tunnel was performed in six patients, while in the remaining four patients, the reconstruction of the PLC was performed with a single femoral tunnel. Six patients had semitendinosus allograft and four had semitendinosus autograft. All patients had the same evaluation and the same rehabilitation protocol. Results Mean follow-up was 27.5 months (range 18-40 months). Mean range of motion (ROM) was 143.5 degrees for flexion (range 135-150 and 0.5 degrees for extension (range $0-3^{\circ}$ ). Three patients showed $1+$ on varus stress test, while on Dial test another three patients showed $10^{\circ}$ reduction of external rotation compared with contra-lateral knee. The mean Lysholm score was 94 points (range 83-100), and the mean International Knee Documentation Committee (IKDC) subjective result was 88.48 (range 74-96.5). Based on Lysholm score, the results were excellent in eight knees and good in two knees. On IKDC evaluation, two patients were grade $\mathrm{A}$ and eight were grade $\mathrm{B}$. No significant difference in clinical results was observed between single and double femoral tunnel. Conclusions Fibular-based technique showed good results in terms of clinical outcome, restoring varus and rotation stability of knees in treatment of chronic isolated PLC injury.
ACL reconstruction and development of knee arthritis: long term clinical and radiographic follow-up and analysis of correlations

\section{Surace, P. Bulgheroni, D. Marcolli, A. Fagetti, P. Cherubino}

Dipartimento di Scienze Chirurgiche Ricostruttive e Tecnologie Avanzate (Varese, ITALY)

Anterior cruciate ligament (ACL) lesion is a frequent pathology in athletes.

Aim of our study is the research and evaluation of clinical and radiographic signs of gonarthrosis after 15 years from ACL reconstruction surgery. Through a comparison with the data retrieved in literature, we try to understand if the surgical treatment of an ACL lesion is able to prevent the arthritis development, besides granting the continuation of sports activity that requests a stable knee joint. We considered 32 patients that underwent ACL reconstruction between 1992 and 1997. All patients were evaluated through clinical and radiological exam between July and September 2009. The instrumental exam was performed through the use of a KT-2000 (Knee Ligament Arthrometer MEDmetric Corp. San Diego, Ca). This tool grants the possibility to measure the anterior shifting of the tibia. To obtain the subjective assessment we used the Lysholm Knee Scale, KOOS and the Tegner Activity Scale.

The radiographic evaluation of the knee was performed trough lower limbs X-rays under full weight, lateral and Rosenberg projections to evaluate the intercondilar notch. The radiographic images were classified using the Ahlback scale and the Kellgren and Lawrence scale. The data obtained undergone statistical analysis with the assistance of a type SPSS software (SPSS 11.0, SPSS Inc., USA).

From the data analysis we gather that all the patients of this study, which were treated for ACL reconstruction, after a mean period of 14 years from surgery, developed a certain level of arthritis. The data obtained from the subjective evaluation, however, show a general satisfaction of the patients for the actual condition of their knee.

The consequence during follow-up of an ACL injury surgically treated is the appearance of degenerative lesions associated to arthritis. At a mean follow-up of 14 years we found the presence of initial arthritis signs that even if they did not compromise the quality of life, they reduced the sports performance of the patients. The age at the time of the injury and the mean follow-up, as long as the time passed from the injury to the surgery do not seem to affect the arthritis development.

We underline the absence of a relationship between the X-ray data and the clinical conditions of the patients. The good clinical conditions do not relate necessarily to absence of arthritis development; viceversa some patients that have pain and functional limitations can have at the X-ray evaluation a good condition of the joint.

\section{ARTHROSCOPY 3}

\section{Avoiding tunnel collisions between fibular collateral ligament and ACL postero-lateral bundle reconstructions}

\section{Camarda $^{1}$, M. D’arienzo ${ }^{1}$, R. F. Laprade ${ }^{2}$}

${ }^{1}$ Department of Orthopaedic and Traumatology, University of Palermo (Palermo, ITALY);

${ }^{2}$ The Steadman Philippon Research Institute (Vail-Colorado, USA) 
Background Double bundle anterior cruciate ligament (DB-ACL) reconstructions are a recognized technical alternative to primary single bundle ACL reconstruction which could restore knee kinematics closer to the normal knee. However, with this procedure, the postero-lateral (PL) ACL tunnel is placed more horizontal and closer to the primary posterolateral corner (PLC) structures. The purpose of our study was to conduct an experimental study using synthetic femurs to evaluate the risk of tunnel collisions of the FCL and PLACL tunnels during a combined FCL and DB-ACL reconstruction.

Material and methods 4 th generation synthetic femurs were utilized. There were eighteen medium and large sized femurs each. A FCL tunnel and a postero-lateral ACL (PL-ACL) bundle tunnel were reamed on each femur. Nine different orientations of the FCL tunnel were created using $20^{\circ}$ intervals in both the coronal and axial planes. 25 and $30 \mathrm{~mm}$ FCL tunnel depths were reamed. Two different orientations of the PL-ACL tunnel were chosen to simulate different PLACL tunnel directions that could be obtained through a low anteromedial (AM) portal. The tunnels of synthetic specimens that didn't have a collision were then filled using an epoxy resin augmented with BaSo4 and radiographic evaluation and CT were performed.

Results The rate of tunnel collision when the FCL tunnel was reamed to a depth of $30 \mathrm{~mm}$ was $75 \%$. The rate of tunnel collision decreased to $69.4 \%$ when the FCL tunnel was reamed to a depth of $25 \mathrm{~mm}$. There was a significantly increased risk of tunnel collision when the FCL tunnel was reamed proximally with coronal angulations of $20^{\circ}$ and $40^{\circ}$. No collisions were noted when the FCL tunnel was reamed parallel to the distal condylar line and with axial angulations of $20^{\circ}$ and $40^{\circ}$. This was observed for both PL-ACL tunnels and femur sizes. Discussion We observed that the risk of collisions between the FCL and PL-ACL Bundle tunnel is high, but it could be avoided by limiting proximal angulations of the FCL tunnel and directing the FCL tunnel on front with an axial angulation of $20^{\circ}$ or $40^{\circ}$. This study provides new insight into tunnel positioning during a combined FCL and DB-ACL reconstruction. Furthermore, the results suggest that a concomitant FCL injury not seem to represent a contraindication to perform a DB-ACL reconstruction as long as the FCL tunnel is performed with no proximal angulation and directed on front with an axial angle of $20^{\circ}$ or $40^{\circ}$.

\section{ACL reconstruction with hamstrings: how different techniques and fixation devices influence bone tunnel enlargement} R. Iorio, A. Vadalà, F. Conteduca, J. Conteduca, G. Argento,
A. Ferretti

Orthopaedic and Traumatology Department, University La Sapienza (Rome, ITALY)

Objective Mechanical factors are thought to be one of the main reasons in determining tunnel enlargement after ACL reconstruction with hamstrings. The purpose of this prospective study was to evaluate how the different surgical techniques may affect the bone tunnel enlargement.

Material and methods Forty-five consecutive patients undergoing ACL reconstruction with the use of autologous doubled semitendinosus and gracilis tendons entered this study. They were randomly assigned to enter group A (In-Out technique, with cortical fixation and Interference screw) and group B (Out-In technique, metal cortical fixation on the femour and tibia). At a mean follow-up of 10 months, all the patients underwent clinical evaluation and a CT scan exam to evaluate the postoperative diameters of both femoral and tibial tunnels.
Results The mean femoral tunnel diameter increased significantly from $9.05 \pm 0.3 \mathrm{~mm}$ (post-operatively) to $10.01 \pm 2.3 \mathrm{~mm}$ (followup) in group A and from $9.04 \pm 0.8 \mathrm{~mm}$ to $9.3 \pm 1,12 \mathrm{~mm}$ in group B. The mean tibial tunnel diameter increased significantly from $9.03 \pm 0.04 \mathrm{~mm}$ to $10.68 \pm 2.5 \mathrm{~mm}$ in group $\mathrm{A}$ and from $9.04 \pm 0.03 \mathrm{~mm}$ to $10 . \pm 0.78 \mathrm{~mm}$ in group B. The mean increase in both femoral and tunnel diameters observed in group A was significantly higher than that observed in group B $(p<0.05)$. Stability evaluated with KT-1000 arthrometer does not significantly differ in the two groups

Conclusions The results of this study suggest that the use of different hardware devices may affect tunnel enlargement after ACL reconstruction with hamstrings. The conclusion can be reached depending on the different stiffness of the devices used and on their backlashes on the tunnel walls, which may favor the bungee jumping or the windshield wiper phenomenons.

\section{Anterior cruciate ligament reconstruction with Zip-Loop femoral fixation}

\author{
G. Lensi, M. Della Monaca, M. Capone, A. Fascetti
}

Ospedale Versilia (Lido di Camaiore, ITALY)

We describe their experience about LCA reconstruction, using ZipLoop device for femoral fixation. The Zip-Loop is used from 2008 at Versilia Hospital.

This new cortical suspension device (Zip-Loop; BIOMET Sport Medicine) has been introduced for fixation of the graft on the femoral side and it can be used with BPTB and with Hamstrings.

We describe the Zip-Loop advantages and features as well as some surgical techniques to make its use easily reproducible and not operator dependent.

\section{Lateral notch sign: comparative evaluation in patients with anterior cruciate ligament lesion}

\author{
M. Ronga ${ }^{1}$, G. Gianazza ${ }^{1}$, E. Paiusco ${ }^{1}$, E.A. Genovese ${ }^{2}$, \\ P. Cherubino ${ }^{1}$ \\ ${ }^{1}$ Department of Orthopaedics and Traumatology and ${ }^{2}$ Department \\ of Radiology, University of Insubria (Varese, ITALY)
}

Objective The lateral notch sign (LNS) is a sulcus of the lateral femur condyle, visible on lateral view X-rays, determined by the impact of the condyle with the postero-superior region of the lateral tibial plate during traumas responsible for ACL rupture. The aim of this study is to evaluate qualitatively and quantitatively the LNS.

Material and methods Eighty consecutive patients affected by ACL tear with a healthy contralateral knee were included in the present study. Both knees were evaluated at X-ray. LNS was measured by tracing the perpendicular to the tangential of the sulcus. Nakauchi classification was used to evaluate the type of LNS. Frequency and depth of LNS was evaluated by two-first-year orthopaedic surgery residents independently. Three evaluations were performed, 1 week from the other, by one of the observers randomly chosen. The results were then correlated to the type of trauma mechanism, presence of meniscal lesions, age, sex and sport practiced by the patient at the time of trauma. 
Results LNS frequency was 36 on 80 patients (45\%) and 34 on 80 patients $(42.5 \%)(p>0.05)$, respectively. Mean depth was $1.3 \mathrm{~mm}$ (range, $0.5-2.9 \mathrm{~mm})$ and $1.2 \mathrm{~mm}$ (range, $0.5-2.9 \mathrm{~mm})(p>0.05)$, respectively. No significant differences were observed in the LNS classification and in the three different evaluations performed by the single operator. LNS was mostly associated to a trauma in valgusextrarotation and to lateral meniscus lesions $(p<0.05)$. No other correlations between LNS and the other variables were observed.

Conclusions LNS is a reliable and easily identifiable indirect radiographic sign of ACL lesion. The association of LNS with traumas in valgus-extrarotation and the positivity for lateral meniscal signs strengthens the hypothesis of a ruptured LCA. The future objective is to complete a long-term study on the same patient population to evaluate possible clinical and radiographic evolutions of the lesion.

\section{ARTHROSCOPY 4}

\section{The chondral lesions: our experience with II generation autologous chondrocyte on three-dimensional collagen scaffold}

\section{S. Giannotti, M. Ghilardi, V. Bottai, G. Dell'Osso, G. Guido}

Orthopaedic Clinic, University of Pisa (Pisa, ITALY)

Objective Spontaneous healing capacity of cartilage damage is low, currently there are several surgical techniques that have as a goal not only to restore long-term joint function without pain but also to promote the formation of healing tissue is as similar as possible to normal cartilage [1]. The premise of the use of autologous chondrocytes is precisely to enable a better quality of tissue healing. Among the various methods of ACI technique proposals from the market and that we have used [2], we examined the cases of arthroscopic implant of II generation chondrocytes with the Novocart 3D system.

Material and methods The Novocart 3D system provides the proliferation of cells on biphasic matrix consisting of a dense layer of collagen fibers perfectly oriented in longitudinal sense, so do not leave blank spaces where the cells could migrate; a large layer "sponge-like. It is precisely this peculiar spongy structure to determine the three-dimensional homogeneous distribution of cells. We treated with this technique 16 patients, 10 male and 6 female with a mean age of 25 years ( $\min 14-\max 41)$. We performed 12 operations on the knee and 4 on the ankle.

Results Patients underwent a clinical evaluation with the card for the Lysholm knee and Ogilvie-Harris to the ankle with a mean follow-up of 16 months resulting in excellent clinical results. In two cases we performed a second-look arthroscopy during which a biopsy of the treated area was performed, according to the ICRS guidelines. The histological results showed good cell regeneration in both cases showing the formation of hyaline cartilage in a patient and the formation of fibrocartilage in the other case.

Discussion and conclusions Depending on the different types of lesions treated these results showed that with the correct information this method can restore a cartilaginous articular surface quality comparable to the anatomy as well as ensuring good clinical and functional results in the medium term and long-term perspective.

\section{References}

1. Saris DBF, Vanlauwe J, Victor J, Haspl M, Bohnsack M, Fortems Y, Vandekerckhove B et al. (2008) Characterized Chondrocyte Implantation Results in Better Structural Repair When Treating Symptomatic Cartilage Defects of the Knee in a Randomized Controlled Trial Versus Microfracture. Am J Sports Med 36:235
2. Kasemkijwattana C, Kesprayura S, Chaipinyo K, Chanlalit C, Chansiri K (2009) Autologous chondrocytes implantation with three-dimensional collagen scaffold. J Med Assoc Thai 92(10):1282-1286

\section{Matrix-induced autologous chondrocyte implantation (MACI): high-resolution-MRI study at medium term follow-up}

M. Ronga $^{1}$, P. Bulgheroni ${ }^{1}$, G. Angeretti ${ }^{2}$, E. A. Genovese ${ }^{2}$, P. Cherubino

${ }^{1}$ Department of Orthopaedics and Traumatology and ${ }^{2}$ Department of Radiology, University of Insubria (Varese, ITALY)

Objective Evaluation of articular cartilage repair after Matrixinduced autologous chondrocyte implantation (MACI) by high-resolution standard MRI and arthro-MRI.

Material and methods Thirty patients with a minimum of 5 years follow-up (mean 67 months, range 60-84 months) were evaluated with a high-resolution standard MRI and arthro-MRI (1.5-T unit, using GE, T2*, spin-echo T1, FatSat fast spin-echo DP and T2weighted sequences). The results were classified according to the grading system described by Marlovits that considered the degree of defect repair in width and length, surface, structure and signal intensity of the repair tissue, and status of the subchondral lamina and bone. A score higher than 50 points (max: 100 points) was assumed as a successful repair. The results were then compared to the most common clinical rating scales (ICRS, Lysholm II, Tegner, Cincinnati) to evaluate the possible correspondence.

Results In 18 patients we observed a complete filling of the defect. Implant integration was evident in 17 cases. Intact surface was found in 18 cases. Arthro-MRI revealed 7 cases of superficial fibrillations and fissures. In 23 implants the signal was isointense and similar to native cartilage. Oedema or cysts were individuated in 9 cases. In 23 patients we recorded an overall value higher than 50 points. Four cases were associated with a clinical failure while 3 cases were not associated.

Conclusions High-resolution MRI provides a useful tool for studying articular cartilage repair tissue. Arthro-MRI can improve the sensibility of the study to detect any clefts between native cartilage and regenerated tissue and any superficial implant fibrillations. It has to be demonstrated if MRI can be predictive of future clinical failures. Only an higher number of cases and long term studies will allow to confirm these observations.

\section{METABOLIC DISORDERS}

\section{Immunohistochemical analysis of the skeletal muscle atrophy}

M. Marinelli, L. Memè, M. Pavan, A. Orazi, L. de Palma

Cattedra di Ortopedia e Traumatologia, Università Politecnica delle Marche, Azienda Ospedaliero-Universitaria, Ospedali R (Ancona, ITALY)

Several pathological conditions can induce skeletal muscle atrophy and seem to share common enzyme pathways. In catabolic states where proteolysis is increased, two genes specific to muscle atrophy, MuRf1 
and MAFbx, are upregulated. These encode ubiquitin ligases, which bind to and mediate ubiquitination of myofibrillar proteins for subsequent degradation during muscle atrophy. Fifteen patients undergoing leg amputation were divided into two groups. Group A included 12 elderly patients (mean age 79 years) amputated for vascular disease (complicated by diabetes in four), chronic osteomyelitis or squamous cell carcinoma. Group B included three car accident victims (mean age 32 years) amputated due to acute arterial insufficiency. Gastrocnemius muscle biopsies were collected for a histochemical and immunohistochemical (anti-MuRf1, anti-MAFbx) study.

Group A specimens showed a decreased cross-sectional fiber area and length, adipose tissue replacement, and MuRf1 and MAFbx immunoreactivity. Muscle cells showed MuRf1 and MAFbx subsarcolemmal immunoreactivity and weak extracellular matrix immunoreactivity. Group B samples exhibited mild muscle structural changes; they did not stain with anti-MuRf1 or anti-MAFbx, and neither did sections showing muscle degeneration and adipose tissue replacement.

Results showed upregulation of MuRf1 and MAFbx in atrophied muscle and support their role as regulatory peptides in various conditions that lead to muscle atrophy. Data suggest that the study of cellular pathways can help identify promising targets for effective new treatments for skeletal muscle atrophy. Clinical Relevance. The treatment of several orthopaedic conditions is complicated by muscle atrophy; potential treatments could be directed to specific sites where these proteins are localized.

\section{Foot alterations in primary hyperparathyroidism. Radiological features}

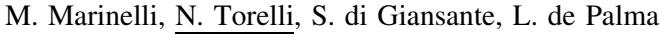

Cattedra di Ortopedia e Traumatologia, Università Politecnica delle Marche, Azienda Ospedaliero-Universitaria. Ospedali R (Ancona, ITALY)

The skeletal involvement of the foot (and of the hand) in primary hyperparathyroidism is precocious and constant.

Some of the radiological features observed in the foot have a diagnostic significance; otherwise other signs are not so characteristic and, if present alone, are not so useful in the diagnostic process.

The aim of this contribution is the description of the very characteristic radiological features of this pathological condition.

The widespread osteoporosis is constant, but also show a very a specific aspect: it is present in different diseases.

Radiographic examination of the feet reveals erosion of the phalangeal tufts and subperiosteal reabsorption.

At the beginning of the reabsorption phenomenon, the radiological feature of the phalanx is very characteristic: some authors use the term "lace" erosion. The progression of the reabsorption phenomenon can lead to the complete loss of the cortical bone. The same reabsorption phenomenon involves the apex of the distal phalanx with different erosion features. The periostial reabsorption is also characteristic of the epiphyseal site.

The subchondral reabsorption is a typical radiological aspect: between areas of osteoporosis of the subchondral bone there are areas of sclerosis that cause chondral suffering and alteration of the articular surface.

Reabsorption phenomenons are also present at the posterior tuberosity of the calcaneus characterised by erosion process, alteration of the bone surface and proliferative reaction.

The so-called "brown tumor" is a peculiar aspect of primary hyperparathyroidism and it can also be detected in the foot.
From a radiological point of view brown tumors are characterized by lytic lesions: single or multiple lesions, with well demarked edges or only in part defined, in a central site or in an eccentric site, with small diameters or so wide to deform and, sometimes, to cause a pathologic fracture.

Finally, in the primary hyperparathyroidism some skeletal features of the foot are characteristic.

Recognizing these radiological signs can be useful to discover the illness and to start a precocious treatment.

\section{HAND AND WRIST 1}

\section{Chondrosarcoma of the hand}

\author{
R. De Vitis, G. Taccardo, S. Serpieri, F. Fanfani, F. Catalano
}

Complesso Integrato Columbus, Policlinico A. Gemelli, Università Cattolica Sacro Cuore (Rome, ITALY)

Objective The cartilagineous tumors represent the $88 \%$ of the hand tumors: among the benign tumors the most frequent is condroma, while among the malignant ones the most frequent is chondrosarcoma.

Hand chondrosarcoma however is very rare, while it is being more common in pelvis or in proximal femur and the humerus.

Material and methods We reviewed 224 cartilagineous tumors of the hand treated by curettage, most described in the literature and in addition our personal histories of three cases: 2 men (3rd and 5th metacarpal bone) and a woman (proximal phalanx of the 2nd finger), mean age 62 years.

Discussion and conclusions The differential diagnosis between chondroma and chondrosarcoma in the hand is very difficult because there are not visible radiographic differences and chondroma in this anatomical district has histological characteristics typical of the low grade chondrosarcoma (elevated cellularity, ample nucleuses, binucleate cells) although the first one is completely benign. In fact, Campanacci affirmed that $10 \%$ of hand chondromas show histological aggressiveness although they result chondromas eventually.

To date the discussion about the origin of hand chondrosarcoma is still open. Some authors affirm that hand chondrosarcomas may develop from a preexisting chondroma, while others recommend great prudence because of no sure observations of malignant transformation of the hand chondroma.

Dahlin specified that the biopsy is not enough to differentiate the chondroma from the chondrosarcoma, in fact, besides the evident atypical aspect in histological examination we have to consider the patient's age, the presence of pain, the possible massive destruction of the bone, the invasion of the soft tissues and the rapid evolution of the lesion. With the present job we introduce our diagnostic-therapeutics considerations on hand chondrosarcoma.

\section{Suggested readings}

1. Kohli K, Lallawmzuali D, Kawatra V, Khurana N (2009) Dedifferentiated chondrosarcoma of the hand: case report. Pathology 41(5):491-494

2. Ennouri K, Charfi H, Baccari S, Tarhouni L, Jaafoura H, Bahri H (2006) Chondrosarcoma of the hand. Chir Main 25(1):40-47

3. Canizares F, Garrido I, Chevreau C, Pinsolle V, Grolleau JL, Chavoin JP (2007) The chondrosarcoma of the hand: review of the literature about a case and a proposition of a decision-making therapeutic tree. Ann Chir Plast Esthet 52(6):609-615 


\section{Thumb arthritis: treatment by hyaluronic acid}

\section{S. Odella, R. Sartore, F. Torretta}

Istituto Ortopedico G. Pini (Milan, ITALY)

Purpose and treatment indications The first carpo metacarpal joint is the second most affected site by arthritis, after the hand distal phalanx joint. To date the surgical treatment is not completely of satisfactory, because of the important limitation of hand function, strength and mobility. Patients affected by symptomatic thumb arthritis have pain irradiating to the tenar region, pinch strength reduction, swelling and joint deformity.

Material and methods In our Operative Unit, from January 2008 to January 2010, 30 patients were treated by hyaluronic acid infiltration (Curavisc Mini), they included 25 female and 5 male affected by thumb arthritis at different stages. Patients underwent fluoroscan guided infiltration every 2 weeks, 5 times consecutively. In one we obtained complete pain remission after only three infiltrations and stopped the therapy. Hyaluronic acid is constituted by polisaccaridis of $\mathrm{N}$-acetil glucosamine and glucuronic acid: elements usually part of synovial liquid and important to maintain normal joint function. The action of hyaluronic acid is the increase of the joint cartilage components synthesis and the reduction of degradation. It also induces an analgesic effect by the inhibition of inflammation and pain receptors [1].

Results The results we could evaluate till now are good as concerning pain. The pathology stage does not influence the results at short term; best results have been obtained in female without heavy activity.

Conclusions This prospective study was aimed at evaluating the efficacy of hyaluronic acid infiltration in pain remission, and in daily activity in case of patients affected by thumb arthritis.

\section{Reference}

1. Altman RD (2003) Status of Hyaluronan Supplementation in therapy in osteoarthritis. Current Rheumatology Reports 5(1):7-14

\section{Our experience in the use of partial scaphoid prostheses APSI}

\section{Zecca, M. Musso, M. Ghezzi}

\section{Istituti Clinici di Perfezionamento, CTO (Milan, ITALY)}

Objective We present the experience of partial replacement of the carpal scaphoid using the prosthesis APSI (Adaptive proximal Scaphoid Implant)

Material and methods Twenty-one patients with carpal scaphoid nonunion or necrosis of the upper pole of the scaphoid following a fracture have been treated with APSI prosthesis by using the access road back. They have been evaluated by three-dimensional CT reconstructions and radiographic study of the radio-carpal joint preoperatively and post-operatively and during periodic remote examinations.

Results The functional recovery of radio-carpal joint and the clinical presence of pain have been evaluated. We have also evaluated the radiocarpal joint angle by a radiographic study and the adaptation of implant through dynamic tests.

Conclusions We describe our experience with the prosthesis APSI, the surgical technique, the clinical indications and the long-term results concerning a rare disease that leads outpatient observation.

\section{HAND AND WRIST 2}

\section{Osteosynthesis-stabilization by bone graft according to Matti-Russe in nonunion of the carpal scaphoid}

\author{
G. Taccardo, R. De Vitis, V. Cilli, F. Fanfani, F. Catalano
}

Complesso Integrato Columbus, Policlinico A. Gemelli, Università Cattolica Sacro Cuore (Rome, ITALY)

Objective The fractures of the scaphoid represent $70 \%$ of the fractures of the carpal bones. Missed consolidation realizes in more than the $90 \%$ of the unstable fractures treated by the conservative treatment, evolving in nonunion. The carpal scaphoid bone, at 6 months from the fracture, loses every stimulus to the spontaneous consolidation and it goes towards a progressive reabsorption and shortening. The missed treatment of a nonunion of the carpal scaphoid, in varying times determines degenerative alterations of the radio-carpal and medio-carpal joints until an arthrosis in advanced phase with initial collapse of the carpus (wrist SNAC). The osteosynthesis-stabilization with bone graft according to Matti-Russe is suitable in the treatment of the carpal scaphoid nonunion up to the initial phases of arthrosis.

Material and methods We used a bony graft of suitable dimensions to restore length and morphology of the scaphoid, press fit installed and not stabilized with a synthesis, that contributes to the consolidation through both the biological stimulus typical of bone grafts and the stabilization of the stumps of nonunion. In the fractures of the scaphoid body (type 2, 3 and 4 of Schernberg) a reabsorption is primarily volar and in the proximal fragment, for which the inveterate nonunion evolves in shortening and bending of the scaphoioid with an adaptative carpal disorder, then lunate bone rotates in extension to the proximal fragment of the scaphoid (DISI). In such cases we also used a butterfly bone graft in which the length of the graft assures the restoration of the length of the scaphoid and the wings assure the correction of the bending filling the volar loss of bony substance.

Discussion and conclusions We expose our considerations concerning the revision of our case histories of carpal scaphoid nenunion treated by bone grafting, with a 5 -year minimum and a 10 -year mean follow-up.

Suggested readings

1. Buijze GA, Doornberg JN, Ham JS, Ring D, Bhandari M, Poolman RW (2010) Surgical compared with conservative treatment for acute nondisplaced or minimally displaced scaphoid fractures: a systematic review and meta-analysis of randomized controlled trials. J Bone Joint Surg Am 92(6):1534-1544

2. Arora R, Lutz M, Zimmermann R, Krappinger D, Niederwanger C, Gabl M (2010) Free vascularised iliac bone graft for recalcitrant avascular nonunion of the scaphoid. J Bone Joint Surg $\mathrm{Br}$ 92(2):224-229

3. Koodziej RK, Blacha J, Bogacz A, Mazurkiewicz T (2006) Longterm outcome of scaphoid nonunion treated by the Matti-Russe operation. Ortop Traumatol Rehabil 8(5):507-512

\section{Tumb arthritis at stage 2: results of the treatment with pyrocarbon prostheses}

\section{S. Odella, R. Sartore}

Istituto Ortopedico G. Pini (Milan, ITALY) 
To this day the thumb arthritis treatment does not recognize a gold standard, we cannot find an obvious correlation between the radiographic stage and the clinical results after surgery [1]. Earp considers pyrocarbon prosthesis a valid solution in case of thumb arthritis at stage 2. Pyrocarbon is a material characterized by an elastic modulus similar to the bone, and by a low friction coefficient; it is considered a valid tool for the treatment of thumb arthritis [2]. The purpose of our study is to evaluate the results of the treatment by using Pyrodisc. In our Operative Unit from January 2007 to January 2009, 24 patients affected by thumb arthritis at stage 2 by Eaton, were operated consecutively. The patients were treated by partial osteotomy of the trapezium and positioning of a pyrocarbon spacer, no dislocations of the first metacarpal bone were observed. The population mean age was 64 years $(\min 51, \max 70)$, in 22 cases patients were female, in 2 cases they were male; patients have been studied prospectively until 2 years from surgery.

One implant was removed although the radiographic analysis showed a correct positioning of the spacer, because the patient complained pain after 6 months from surgery.

We found that the remission of the pain at the trapeziometacarpal joint and at the donor site (radial flexor carpis) was complete only after 6 months from surgery. The pain was reduced in intensity e durability if we executed the suture of the TMC joint capsule by keeping the thumb in adduction after inserting the spacer.

\section{References}

1. Earp BE. Treatment of advanced CMC joint disease: trapeziectomy and implant arthroplasty (silastic metal synthetic allograft)

2. Perrone V (2009) Gli impianti protesici e gli spaziatori in pirocarbonio nella chirurgia della mano: risultati preliminari. Minerva Ortopedica e Traumatologica 60(1):9-19

\section{FOOT AND ANKLE 1}

\section{The use of PLLA fixation in corrective foot surgery}

\author{
L. Gaiani, R. Bertelli, M. Palmonari \\ Ospedale Civile di Imola (Imola, ITALY)
}

Introduction Polylactic acid is a synthetic polymer of lactic acid which belongs to the category of absorbable materials. Orthopaedic surgery lends itself to numerous applications and particularly in foot surgery.

Material and methods In the period from November 2008 to September 2009 were included in this study 10 patients, 2 male and 8 female, mean age 56 years (maximum 68 years, minimum 45 years). In all patients triplanar osteotomy and correction of little toes by using wires $2 \mathrm{~mm}$ polylactic acid (Arthrex, Inc. Naples, FL 34108-1945, USA) was performed. The average follow-up period was 14 months (maximum 22 months, minimum 7 months). Patient evaluations were done using the score of American Orthopedic Foot and Ankle Society (AOFAS). Results The mean AOFAS score improved from 24 to 84 points. The intermetatarsal angle (IM) has increased from an average of $13.2^{\circ}$ preoperatively to $8.7^{\circ}$ postoperatively while the angle of metatarsophalangeal (MTP) has increased from a preoperative average of $26.8^{\circ}$ to a postoperative average of $14.4^{\circ}$. The $90 \%$ of the patients was satisfied with the result, $10 \%$ was not pleased with the aesthetic result but not with the functional. We did not have relapses or restrictions in activities of daily living.

Discussion We believe the benefits of this surgical technique are many: no adverse reaction, no residue of the plant and an excellent stability of the osteotomy that provides a lasting correction.

\section{FOOT AND ANKLE 2}

\section{Arthropaties of the tibiotarsic joint in adults: a comparison between arthroplasty and arthrodesis}

\author{
M. Massobrio, F. Necci, G. Antonietti, P. Albanese, C. Esposito
}

(Rome, ITALY)

Introduction We compared the indications and the results of the treatment of the tibiotarsic joint degenerative pathology, treated either with arthroplasty or arthrodesis surgery.

Material and methods We studied 20 patients with degenerative and post-traumatic deformities surgically treated from 2004 to 2009 . We created 2 groups depending on the performed treatment. The patients $(\mathrm{n}=10)$ treated with an ankle prosthesis had a mean age of 53 (min 41, $\max 73)$. The patients $(\mathrm{n}=10)$ treated with a tibiotarsic arthrodesis had a mean age of $61(\min 47, \max 74)$. Indications for the prosthesis were: medium grade talus degeneration, preserved joint range of motion, absence of tibial or calcanear deformities, absence of instability [1]. Indications for the arthrodesis were: impossibility of prosthesis application, deformity type, results of a previous tibiotarsic artrodesis [2].

Results In both groups we achieved the deformity correction and the restoration of the limb function (AOFAS Table). In all group 1 patients (arthroprosthesis) pain was immediately relieved, and they were able to deambulate normally within 2 months after surgery. The radiographic criteria to establish a correct positioning of the prosthesis were: in $\mathrm{AP}$ varus $\max 3^{\circ}$, valgus $\max 7^{\circ}$. In LL, correspondence of the tibial and talar axis. One year after surgery, the AOFAS mean score was 80 . In group 2 (retrograde nail) the recovery of normal deambulation was achieved 3 months after surgery, the residual limb hypometry ranged from $1 \mathrm{~cm}$ to $2.5 \mathrm{~cm}$. The absence of pain was immediate. The AOFAS mean score was 74 .

Discussion and conclusions The indications for the arthoprosthesis surgery are limited to the cases where the degeneration is not severe, due to the necessity of a sufficient talus bone stock. The tibiotarsic arthrodesis can be performed independently from the talus bone stock. The use of sagittal screws passing through the calcaneus and the talus has widened its application to deformities that previously could only be treated with external fixation.

\section{References}

1. De Orio JK, Easley ME (2008) Total Ankle Arthroplasty. AAOS Instructional Course Lectures 57:383-413

2. Hopgood P, Kumar R, Wood PLR (2006) Ankle Arthrodesis for failed total ankle replacement JBJS (Br) 88-B:1032-1038

\section{Short-medium term clinical and radiographic results of the total ankle prosthesis "BOX"}

\author{
C. Pasquali ${ }^{1}$, S. Moalli ${ }^{1}$, C. Manzini ${ }^{2}$, R. Pelucchi ${ }^{2}$ \\ ${ }^{1}$ Department of Orthopaedic Surgery, Circolo Fondazione Macchi \\ Hospital (Luino, ITALY); \\ ${ }^{2}$ Department of Orthopaedic Surgery, Carate Hospital (Vimercate, \\ Giussano, ITALY)
}

Objective Evaluate clinical and radiographic results of a new total ankle prosthesis design which is capable of maintaining complete congruence at the two articulating surfaces of the meniscal bearing over the entire motion arc and restore the characteristic original pattern of ligament tensioning [1]. The use of ankle arthroplasty as an 
alternative to ankle fusion is getting more and more frequent; indications are primary or post-traumatic osteoarthritic patients with a low functional demand, usually over 50 years of age, rheumatoid arthritic patients.

Contraindications are severe morphological defects of the ankle, significant osteoporosis or osteonecrosis, severe vascular or neurological disorders.

Material and methods From January 2007 to November 2009 the BOX prosthesis was implanted in 15 patients, 8 women and 7 men, by two surgeons. The mean patients' age at surgery was 57 years (28-79). We performed ankle prosthesis in a patient with nonunion of the ankle arthrodesis. The main follow-up is 18 months (3-35). The AOFAS score was produced by one physician from the staff of the surgeon at each of the following follow-up: pre-operatively, 3, 6, 12 months post-operatively and yearly. All the patients underwent standard radiography of the ankle, lateral and frontal views, during the follow-up to analyse radiolucency in five adjacent areas under the talar component and in five over the tibial component.

Results The main AOFAS score increase at follow-up is 51 (20-70), the main range of motion increase is $25^{\circ}(10-40)$. One complication in wound healing was solved after 3 months. We observed a radiolucency in a patient's radiography and an increased uptake according to Radionuclide Bone Scan after 2 years, despite of this the AOFAS score increased at the 2 years follow-up. After 6 months every patient was satisfied with his clinical outcome.

Conclusions These results are good even if they are preliminary and the number of cases are few, we must pay attention to select patient candidates for this surgery.

Reference

1. Giannini S, Romagnoli M, O’Connor J, Malerba F, Leardini A (2010) Total Ankle replacement Compatible With Ligament Function Produces Mobility, Good Clinical Scores, and Low Complication Rates. Clin Orthop Relat Res DOI 10.1007/s1-1999-010-1432-3

\section{FOOT AND ANKLE 3}

\section{Surgical treatment of congenital brachymetatarsia using one-stage metatarsal lengthening by allograft interposition}

S. Giannini, C. Faldini, S. Pagkrati, M. T. Miscione, D. Leonetti, M. Nanni, F. Acri

\section{Istituto Ortopedico Rizzoli (Bologna, ITALY)}

Objective Congenital brachymetatarsia consists of shortness of a metatarsal bone, and can be surgically corrected by callus distraction or one-stage lengthening using bone graft. We wondered whether one-stage metatarsal lengthening using metatarsal homologous bone graft could improve forefoot function, lead to metatarsal fusion, restore metatarsal parabola, and improve cosmetic appearance.

Material and methods Fifty metatarsals in 29 patients (41 feet) were lengthened. Surgery consisted of a transverse proximal osteotomy of the metatarsal shaft, and interposition of a metatarsal homologous bone graft (of average $13 \mathrm{~mm}$ long), fixed with an intramedullary Kwire. Minimum follow-up was 3 years (range, 3-11 years).

Results The mean preoperative AOFAS score was 37 (range, 28-53 points), and postoperatively was 88 (range, 74-96 points), with an average improvement of 51 points. Bone union was achieved in all cases. Radiographically, the mean gain in length was $13 \mathrm{~mm}$ (range, $10-15 \mathrm{~mm}$ ), and the main percentage increase was $23 \%$. All patients were satisfied of the cosmetic outcome.
Conclusions One-stage metatarsal lengthening using interposition of metatarsal homologous bone graft is an effective technique, it has low morbidity for the patient, it is rapid and inexpensive with limited complications, it restores a normal forefoot anatomy with good functional, radiographic and cosmetic results.

\section{Dynamic absorbable percutaneous repair of the ruptured Achilles tendon}

\author{
R. Bertelli, L. Gaiani, M. Palmonari
}

Ospedale Civile di Imola (Imola, ITALY)

We have modified the traditional percutaneous repair of the ruptured Achilles tendon so to obtain a lower rate of complications than in open repair, a low rate of rerupture and an early mobilization and return to full weight bearing. We reviewed 50 patients (32 male and 18 female). The repair was carried out using 10 micro-incisions, 5 laterals and 5 medial to the posterior aspects of the tendon with absorbable suture n. 1 (Vicryl, Ethicon, Edinburgh, UK). We used one suture through the four proximal incisions in an 8 shape and one suture trough the four distal as well. We have named this technique "dynamic percutaneous suture" (DPS). The patients were assessed according to the criteria established by the clinical AOFAS rating score. No rerupture or sural nerve damages were observed. In all the treated patients the results obtained were rated from good to excellent. The absorbable suture permits what we call a "dynamic" healing of the tendon, through an "elastic" fixation of the two stumps, as in the healing of a fractured long bone treated with a dynamic nail fixation. We so obtained a short immobilization time and an early full motion and weight bearing.

\section{Clinical and instrumental evaluation of Achilles tendon rupture sutures: semi-percutaneous versus open technique}

D. Munegato $^{1}$, G. Gridavilla ${ }^{1}$, D. Gaddi ${ }^{1}$, M. Bigoni ${ }^{1,2}$, G. Zatti $^{1,2}$

${ }^{1}$ AO San Gerardo (Monza, ITALY);

${ }^{2}$ Università degli Studi di Milano-Bicocca (Milan, ITALY)

Background Subcutaneous rupture of Achilles tendon is a frequent lesion and it has increased during the past decade. Despite its high incidence, the optimal management of Achilles tendon ruptures remains a topic of active debate.

Aim of the study A retrospective comparison between the classic open technique and the semi-percutaneous Ma \& Griffith mod. Osaka technique.

Material and methods A consecutive series of 32 acute Achilles tendon rupture in 29 patients ( 3 bilateral) were treated at our Institution between March 2005 and May 2007; 15 interventions were performed with the open technique (Group A), and 17 with semipercutaneous technique (Group B).

Results The mean follow-up was 15 months in the group B and 25 months in Group A. ATRS score was 90.07 in group A and 92.71 in group B $(p>0.05)$. The mean width of the operated Achilles tendon were $7.67 \mathrm{~mm}$ thicker than the contralateral in group A and $4.07 \mathrm{~mm}$ in Group B $(p<0.05)$. Isokinetic peak force measurement of ankle plantar flexion showed an average loss of $17.25 \%$ in group A versus $8.21 \%$ in group B of the calf muscle strength compared to the normal side $(p<0.05)$. The isokinetic peak force measurement of 
ankle dorsi-flexion were on average reduced of $8.68 \%$ in group A versus $7.21 \%$ in group $\mathrm{B}$. The calf circumference was $23.75 \mathrm{~mm}$ on average reduced in Group A and $10 \mathrm{~mm}$ in Group B related to the contralateral leg $(p<0.05)$. The Patient Satisfaction Score was 96.76 in Group B and 88.67 in Group A $(p<0.01)$ As complications we noted one deep and one superficial infection in group A and one late heel ulcer in group B; one sural nerve lesion in group A.

Conclusions This technique permits to obtain better results in terms of functional scores and subjective satisfaction and minimizes the risk of local complications. Statistically significant advantages in terms of muscles mass and strength recovery of plantar-flexion are proved.

\section{Hypothyroidism: a new risk factor for hallux valgus}

A. Marinozzi, N. Martinelli, E. Franceschetti, B. Zampogna, F. Cancilleri, V. Denaro

Università Campus Bio-Medico (Rome, ITALY)

Objective Hypothyroidism is a dysfunction of thyroid gland due to decreased secretion of the two thyroid hormones thyroxine and triiodothyronine. This pathological condition often follows an autoimmune process, and can cause a progressive loss of thyroid tissue. The aim of our study is to determine the relationship between hypothyroidism and valgus hallux. The prevalence of hypothyroidism in people aged over 65 is estimated to be around 2 and $5 \%$ in the general population.

Material and methods A number of 704 patients was studied (39 men and 665 women, mean age: 62.24 years, range 23-80). These patients had undergone osteotomy of the first metatarsal between 1-1-2000 and 1-9-2009 for valgus deformity of the hallux. Subsequently, patients were divided into two groups, patients with and without hypothyroidism, based on FT3, FT4 and TSH values.

Results A condition of hypothyroidism has been detected in 137 patients out of 704 (19.46\%); among 137 hypothyroid patients, hallux valgus was found in 158 feet (20 recurrences). In 567 patients out of 704, which were not found to be hypothyroid referring to FT3, FT4 and TSH levels, hallux valgus was found in 589 feet (18 recurrences). Conclusions Accordingly, we can assert that there is a relationship between the hypothyroidism and hallux valgus. The rate of hypothyroidism among patients with hallux valgus reached the value of $19.4 \%$, while among the general population it stands at more than $5 \%$. Moreover, an increased rate of recurrences was detected among hypothyroid patients. 